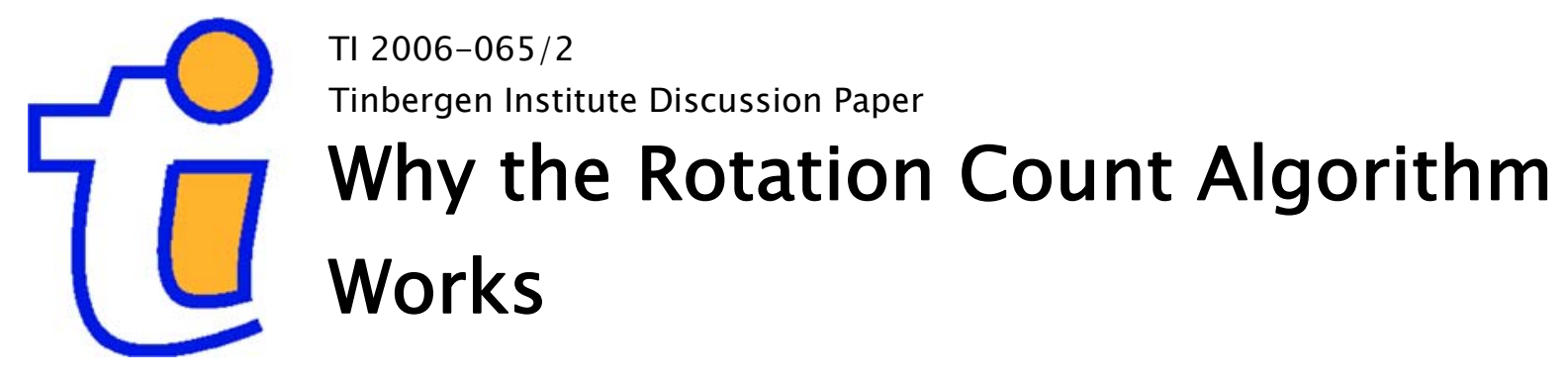

Roger Lord ${ }^{1}$

Christian Kah/2

' Erasmus Universiteit Rotterdam, Rabobank International, and Tinbergen Institute;

2 University of Wuppertal, Germany, and ABN AMRO, London, UK. 


\section{Tinbergen Institute}

The Tinbergen Institute is the institute for economic research of the Erasmus Universiteit Rotterdam, Universiteit van Amsterdam, and Vrije Universiteit Amsterdam.

Tinbergen Institute Amsterdam

Roetersstraat 31

1018 WB Amsterdam

The Netherlands

Tel.: $\quad+31(0) 205513500$

Fax: $\quad+31(0) 205513555$

Tinbergen Institute Rotterdam

Burg. Oudlaan 50

3062 PA Rotterdam

The Netherlands

Tel.: $\quad+31(0) 104088900$

Fax: $\quad+31(0) 104089031$

Most TI discussion papers can be downloaded at http:/ /www.tinbergen.nl. 


\title{
Why the rotation count algorithm works
}

\author{
Roger Lord ${ }^{1}$ \\ Christian Kahl ${ }^{2}$ \\ First version: October $18^{\text {th }}, 2005$ \\ This version: July $17^{\text {th }}$, 2006
}

\begin{abstract}
The characteristic functions of many affine jump-diffusion models, such as Heston's stochastic volatility model and all of its extensions, involve multivalued functions such as the complex logarithm. If we restrict the logarithm to its principal branch, as is done in most software packages, the characteristic function can become discontinuous, leading to completely wrong option prices if options are priced by Fourier inversion. In this paper we prove under non-restrictive conditions on the parameters that the rotation count algorithm of Kahl and Jäckel chooses the correct branch of the complex logarithm. Under the same restrictions we prove that in an alternative formulation of the characteristic function the principal branch is the correct one. Seen as this formulation is easier to implement and numerically more stable than Heston's formulation, it should be the preferred one. The remainder of this paper shows how complex discontinuities can be avoided in the Schöbel-Zhu model and the exact simulation algorithm of the Heston model, recently proposed by Broadie and Kaya. Finally, we show that Matytsin's SVJJ model has a closed-form characteristic function, though the complex discontinuities that arise there due to the branch switching of the exponential integral cannot be avoided under all circumstances.
\end{abstract}

Keywords: Complex logarithm, affine jump-diffusion, stochastic volatility, Heston, characteristic function, moment stability, option pricing.

AMS Classification: 60E10, 91B28.

JEL Classification: C63, G13.

The first author would like to express his gratitude to the Chair of Applied Mathematics / Numerical Analysis at the University of Wuppertal for their hospitality at his visit there. The authors would like to thank Mike Giles for a stimulating discussion in an early stage of this paper. We are grateful to seminar participants at Rabobank International and the Finance mini-symposium at the $42^{\text {nd }}$ Dutch Mathematical Congress for comments.

${ }^{1}$ Erasmus University Rotterdam, Econometric Institute and Tinbergen Institute, P.O. Box 1738, 3000 DR Rotterdam, The Netherlands (e-mail: lord@few.eur.nl, Tel. +31-(0)10-4088935) and Modelling and Research (UC-R-355), Rabobank International, P.O. Box 17100, 3500 HG Utrecht, The Netherlands (e-mail: roger.lord@rabobank.com, Tel.+31-(0)30-2166566).

2 Faculty of Mathematics and Science, Department of Mathematics, Chair of Applied Mathematics / Numerical Analysis, University of Wuppertal, Gaußstraße 20, Wuppertal, D-42119, Germany, and Quantitative Analytics Group, ABN AMRO, 250 Bishopsgate, London EC2M 4AA, UK. 


\section{Introduction}

In this paper we will analyse the complex discontinuities one finds when evaluating the characteristic function of several popular option pricing models, in particular the seminal stochastic volatility model of Heston [1993]. Since the initial breakthrough by Heston a whole range of models have appeared that allow for closed-form characteristic functions. In these models, prices of European options can be calculated semi-analytically by means of Fourier inversion. This approach has in recent years been refined by Carr and Madan [1999], Lewis [2001] and Lee [2004] whose combined works show that if a model has a characteristic function, the forward price of e.g. a European call on an underlying asset S can be written as:

$$
\mathbb{E}\left[(\mathrm{S}(\mathrm{T})-\mathrm{K})^{+}\right]=\mathrm{R}_{\alpha}(\mathrm{F}, \mathrm{K})+\frac{1}{\pi} \operatorname{Re} \int_{0}^{\infty} \mathrm{e}^{-\mathrm{i}(\mathrm{v}-\mathrm{i} \alpha) \mathrm{k}} \frac{\phi(\mathrm{v}-\mathrm{i}(\alpha+1))}{-(\mathrm{v}-\mathrm{i}(\alpha+1))(\mathrm{v}-\mathrm{i} \alpha)} \mathrm{dv}
$$

where $\phi$ is the extended characteristic function of the log-stock price, $\phi(u)=\mathbb{E}\left[\mathrm{e}^{\mathrm{iu} \ln \mathrm{S}(\mathrm{T})}\right]$, and:

$$
\mathrm{R}_{\alpha}(\mathrm{F}, \mathrm{K})=\mathrm{F} \cdot 1_{[\alpha \leq 0]}-\mathrm{K} \cdot 1_{[\alpha \leq-1]}-\frac{1}{2}\left(\mathrm{~F} \cdot 1_{[\alpha=0]}-\mathrm{K} \cdot 1_{[\alpha=-1]}\right)
$$

is a residue term, arising from the poles of the integrand in (1). This representation only holds for values of $\alpha$ satisfying $\phi(-\mathrm{i}(\alpha+1))<\infty$, i.e. for those values of $\alpha$ where the $(\alpha+1)^{\text {th }}$ moment of S(T) is finite. In theory, the option price is independent of the parameter $\alpha$; in practice however, $\alpha$ affects the behaviour of the integrand and choosing the right value for it is crucial. In a recent paper of ours, Lord and Kahl [2006], we outline the optimal choice of $\alpha$.

If we use equations (1)-(2) to evaluate the option price, discontinuities in a characteristic function will clearly lead to discontinuities in the integrand of (1). In turn, this will produce completely wrong option prices, as we will see shortly. The problem at hand is not unique to option pricing, but will occur in any application where one requires an evaluation of a characteristic function. Another example of this is the calculation of density functions via inversion of the characteristic function, see the exact simulation algorithm of Broadie and Kaya [2006] for an application of this in the Heston model.

To fix ideas, we will focus on the Heston stochastic volatility model throughout this paper, though we explore other models in the penultimate section. In the following section we discuss the Heston model and the discontinuities that arise in its characteristic function due to the complex logarithm present in it. Moment stability in the Heston model will also be briefly touched upon, as results from this realm are intimately connected to proofs in later sections. Two formulations of the characteristic function will be presented. The first, which is the original formulation of Heston [1993], is known to suffer from discontinuities when the complex logarithm is restricted to its principal branch. This has been known since Schöbel and Zhu [1999], who proposed an ad-hoc workaround, by letting the integration algorithm used to calculate (1) pick up any discontinuities and correct for them. As this is not foolproof, Kahl and Jäckel [2005a] considered the same problem recently and came up with the rotation count algorithm, an easily implementable algorithm that keeps the complex logarithm in the Heston model continuous. Though the algorithm seems to work perfectly well, a formal proof is required as one does not want to be caught by a counterexample. Section 3 presents this proof for all practically relevant parameter values. Section 4 deals with the second formulation presented in section 2, which is less widespread, but has appeared in e.g. Duffie, Pan and Singleton [2000], Schoutens, Simons and Tistaert [2004] and Gatheral [2005]. It turns out that in this formulation, at least for the same 
range of parameter values considered in section 3, no complex discontinuities will arise if the complex logarithm is restricted to its principal branch. This formulation should therefore be preferred over the first, as it fully obviates the need of the rotation count algorithm. Indeed, we show that if the rotation count algorithm works, the second formulation will not have any complex discontinuities, and vice versa. Finally, in section 5 we consider other models with similar problems and show how to avoid the complex discontinuities there. The models considered are the stochastic volatility models of Schöbel and Zhu [1999] and Matytsin [1999], as well as the recent exact simulation algorithm that Broadie and Kaya [2006] developed for the Heston model and extensions thereof. Section 6 concludes.

\section{Complex discontinuities in the Heston model}

In this section we will first derive the characteristic function of the underlying asset in the Heston model. In the second subsection we discuss the potential complex discontinuities that are present in the Heston model, and present several examples of the impact this could have on option prices. Finally, we touch upon the subject of moment stability. Though this topic has been discussed at great length in the excellent paper by Andersen and Piterbarg [2004], there is an intimate link between moment stability and the proofs in later sections, as we will see.

\subsection{Derivation of the characteristic function}

Under the risk-neutral pricing measure the Heston stochastic volatility model is specified by the following set of stochastic differential equations:

$$
\begin{aligned}
& d S(t)=\mu(t) S(t) d t+\sqrt{v(t)} S(t) d W_{S}(t) \\
& d v(t)=-\kappa(v(t)-\theta) d t+\omega \sqrt{v(t)} d W_{v}(t)
\end{aligned}
$$

where the Brownian motions satisfy $\mathrm{dW}_{\mathrm{S}}(\mathrm{t}) \cdot \mathrm{dW}_{\mathrm{v}}(\mathrm{t})=\rho \mathrm{dt}$. The underlying asset $\mathrm{S}$ has a stochastic variance $\mathrm{v}$, which is modelled as a mean-reverting square root process. The parameter $\kappa$ is the rate of mean reversion of the variance, $\theta$ is the long-term level of variance and $\omega$ is the volatility of variance. Finally, the drift $\mu(\mathrm{t})$ is used to fit to the forward curve of the underlying. We make the following assumption on the parameters:

\section{Assumption:}

$$
\kappa>0, \omega>0,|\rho|<1
$$

If $\omega=0$, the model in (3) collapses to the Black-Scholes model with a time-dependent volatility. Similarly, if $|\rho|=1$, the model is a special case of the local volatility model. The assumption that $\kappa>0$ is not essential, though it will facilitate the analysis. Though the Heston model as postulated here is typically used for asset classes such as equity and foreign exchange, the mean-reverting square root process can be used as a stochastic volatility driver in any asset class, see e.g. Andersen and Andreasen [2002] and Andersen and Brotherton-Ratcliffe [2005] for applications in an interest rate context, and Mercurio and Moreni [2006] for an inflation context.

Although the model in (3) is not affine in the underlying asset $\mathrm{S}$ and the stochastic variance $\mathrm{v}$, it is affine in $\ln \mathrm{S}$ and v. Furthermore, note that the only time-inhomogeneous part of the model is the forward curve of the underlying asset. Hence, following the analysis of Duffie et al. [2000] 
we know that the characteristic function of the logarithm of the underlying will be exponentially affine in the logarithm of the forward, and the stochastic variance:

$$
\phi(u)=\mathbb{E}\left[e^{i u \ln s(T)}\right]=\exp \left(i u f+A(u, \tau)+B_{v}(u, \tau) \cdot v(0)\right)
$$

Here $\mathrm{u} \in \mathbb{C}$ and $\mathrm{f}$ is shorthand for $\ln \mathrm{F}(\mathrm{T})$, the logarithm of the forward price. The functions $\mathrm{A}$ and $B_{v}$ satisfy the following system of ODEs:

$$
\begin{aligned}
\frac{d B_{v}}{d \tau} & =\hat{\alpha}(u)-\beta(u) B_{v}+\gamma B_{v}^{2} \\
\frac{d A}{d \tau} & =\kappa \theta B_{v}
\end{aligned}
$$

subject to the initial conditions $\mathrm{A}(\mathrm{u}, 0)=0$ and $\mathrm{B}(\mathrm{u}, 0)=0$. The auxiliary variables we introduced are $\hat{\alpha}(\mathrm{u})=-\frac{1}{2} \mathrm{u}(\mathrm{i}+\mathrm{u}), \beta(\mathrm{u})=\kappa-\rho \omega u \mathrm{i}$ and $\gamma=\frac{1}{2} \omega^{2}$. Recasting the first Riccati equation as:

$$
\frac{\mathrm{dB}_{\mathrm{v}}}{\mathrm{d} \tau}=\gamma\left(\mathrm{B}_{\mathrm{v}}-\mathrm{a}\right)\left(\mathrm{B}_{\mathrm{v}}-\mathrm{b}\right)
$$

immediately leads to the following solution:

$$
\mathrm{B}_{\mathrm{v}}(\mathrm{u}, \tau)=\mathrm{ab} \frac{1-\mathrm{e}^{(\mathrm{b}-\mathrm{a}) \gamma \tau}}{\mathrm{a}-\mathrm{be}^{(\mathrm{b}-\mathrm{a}) \gamma \tau}}
$$

The roots of the Riccati equation for $B_{v}$ are $a=(\beta+D) / \omega^{2}$ and $b=(\beta-D) / \omega^{2}$ with $D(u)=\sqrt{\beta(u)^{2}-4 \hat{\alpha}(u) \gamma}$. The solution for $B_{v}$ thus equals:

$$
B_{v}(u, \tau)=\frac{\beta(u)-D(u)}{\omega^{2}} \frac{1-e^{-D(u) \tau}}{1-G(u) e^{-D(u) \tau}}
$$

where $G(u)=\frac{\beta(u)-D(u)}{\beta(u)+D(u)}$ represents the ratio of the two roots $b$ and a. This quantity will play a great role in the remainder of the paper. The solution to A now follows from:

$$
\begin{aligned}
\int_{0}^{\tau} B_{v}(s) d s & =\frac{\beta-D}{\omega^{2}} \int_{0}^{\tau} \frac{1-e^{-D s}}{1-G e^{-D s}} d s=\frac{\beta-D}{\omega^{2} D} \int_{G}^{G e^{-D \tau}} \frac{z / G-1}{z(1-z)} d z \\
& =\frac{\beta-D}{\omega^{2} D} \cdot\left[\frac{(G-1) \ln (\mathrm{z}-1)-G \ln \mathrm{z}}{\mathrm{G}}\right]_{\mathrm{z}=\mathrm{G}}^{\mathrm{z}=\mathrm{Ge} \mathrm{e}^{-\mathrm{D} \tau}} \\
& =\omega^{-2}\left((\beta-\mathrm{D}) \tau-2 \ln \left(\frac{\mathrm{Ge}}{\mathrm{G}-1}\right)\right)
\end{aligned}
$$

Clearly there are innumerous ways of writing the characteristic function, and over the years many different formulations have been used. It turns out to be fundamentally important which 
formulation one uses for the function $\mathrm{A}(\mathrm{u}, \tau)$, in particular what one keeps under the logarithm in (10) and what one takes out of it. The formulation we have now derived is what we will refer to as the second formulation, as it is different to the original formulation in Heston [1993]. This formulation appears in e.g. Duffie, Pan and Singleton [2000] $]^{3}$, Schoutens, Simons and Tistaert [2004] and Gatheral [2005]:

\section{Formulation 2:}

$$
\mathrm{A}(\mathrm{u}, \tau)=\kappa \theta \omega^{-2}\left((\beta(\mathrm{u})-\mathrm{D}(\mathrm{u})) \tau-2 \ln \psi_{2}(\mathrm{u}, \tau)\right), \quad \psi_{2}(\mathrm{u}, \tau)=\frac{\mathrm{G}(\mathrm{u}) \mathrm{e}^{-\mathrm{D}(\mathrm{u}) \tau}-1}{\mathrm{G}(\mathrm{u})-1}
$$

The first formulation is the original one used by Heston, and also appears in e.g. the articles of Lee [2004] and Kahl and Jäckel [2005a]:

\section{Formulation 1:}

$$
\mathrm{A}(\mathrm{u}, \tau)=\kappa \theta \omega^{-2}\left((\beta(\mathrm{u})+\mathrm{D}(\mathrm{u})) \tau-2 \ln \psi_{1}(\mathrm{u}, \tau)\right), \quad \psi_{1}(\mathrm{u}, \tau)=\frac{\mathrm{c}(\mathrm{u}) \mathrm{e}^{\mathrm{D}(\mathrm{u}) \tau}-1}{\mathrm{c}(\mathrm{u})-1}
$$

where we introduced $\mathrm{c}(\mathrm{u})=1 / \mathrm{G}(\mathrm{u})$. Though both formulations are algebraically equivalent, it is well-known that formulation 1 causes discontinuities when the principal branch of the complex logarithm is used, whereas for formulation 2 this turns out not to be the case. As an example of yet another formulation we mention Zhu [2000]. As this formulation too causes discontinuities when the principal branch of the logarithm is used, we restrict ourselves to these two formulations. The next subsection discusses the complex discontinuities caused by formulation 1 .

\subsection{Complex discontinuities}

An easy way to avoid any complex discontinuities is to integrate the ODEs in (6) numerically, as this would automatically lead to the correct and continuous solution. A comparative advantage of the Heston model is however that it has a closed-form characteristic function, something which decisively reduces the computational effort and would be forsaken if we proceeded in this way. From a computational point of view it is therefore certainly worthwhile to investigate how to avoid discontinuities in the closed-form solution.

By taking a closer look at the characteristic function, it is clear that two multivalued functions are present, both of which could cause complex discontinuities. The first candidate is the square root used in $\mathrm{D}(\mathrm{u})$. It turns out that the characteristic function is even in $\mathrm{D}$, so that we will from hereon use the common convention that the real part of the square root is nonnegative. The complex logarithm used in (11) and (12) is the second candidate. Let us recall that the logarithm of a complex variable $\mathrm{z}$ can be written as:

$$
\ln \mathrm{z}=\ln |\mathrm{z}|+\mathrm{i}(\arg (\mathrm{z})+2 \pi \mathrm{n})
$$

where $\arg (\mathrm{z})$ is the argument of the complex number, $|\mathrm{z}|$ is its radius and $\mathrm{n} \in \mathbb{Z}$. A typical choice used by most software packages is to restrict the logarithm to its principal branch, by letting $\arg (\mathrm{z})^{4}$ be the principal argument, $\arg (\mathrm{z}) \in[-\pi, \pi)$, and setting $\mathrm{n}=0$. In this case the branch cut of

\footnotetext{
${ }^{3}$ Although our formulation appears slightly different than that of Duffie et al., the term under the logarithm is actually equivalent.

${ }^{4}$ In the remainder $\arg (\mathrm{z})$ will denote the principal argument of $\mathrm{z}$.
} 
the complex logarithm is $(-\infty, 0]$, and the complex logarithm is discontinuous along it. It is welldocumented that by restricting the complex logarithm to its principal branch formulation 1 will yield discontinuities in the characteristic function and hence in the option price. For any set of parameters, unless of course $2 \kappa \theta / \omega^{2} \in \mathbb{N}$, problems will arise if $\tau$ is sufficiently large, and in fact the number of discontinuities will grow with the time to maturity. A first mention of this problem was made by Schöbel and Zhu [1999], who encountered the same problem in the implementation of their own stochastic volatility model. They mention: "Therefore we implemented our formula carefully keeping track of the complex logarithm along the integration path. This leads to a smooth CF...”. It seems that over the past years this approach was, and perhaps still is, best market practice when it comes to the implementation of stochastic volatility models. The problem with this approach is that it requires a very fine integration grid in order to be sure that no discontinuities can arise. Even then, one is not sure that the integration routine has singled out and corrected for all discontinuities, as one does not know a priori how many discontinuities there are.

A significant improvement on this ad-hoc approach has been made by Kahl and Jäckel [2005a], who came up with the rotation count algorithm. This algorithm ensures that the principal argument of $\psi_{1}$ in (12) is continuous. Most importantly, this algorithm is easily implementable and allows for any numerical integration scheme to be used to evaluate the option price in (1), hereby opening up myriads of possibilities for improving the efficiency of implementations of stochastic volatility models. We return to this algorithm in section 3.

Though Kahl and Jäckel have in their paper documented extensively what can and will go wrong if one uses the principal argument of the complex logarithm in conjunction with formulation 1, it is good to stress this again in a realistic example. For this we take the parameters that Duffie et al. [2000] implied from market data of S\&P500 index options. The left panel shows

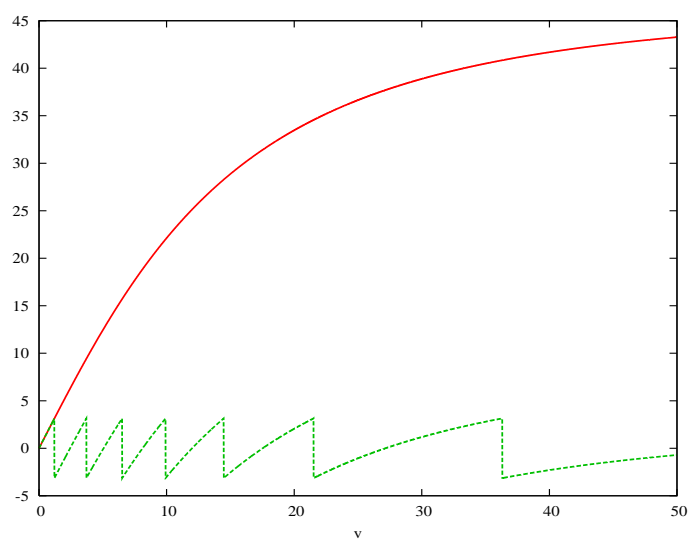

(A)

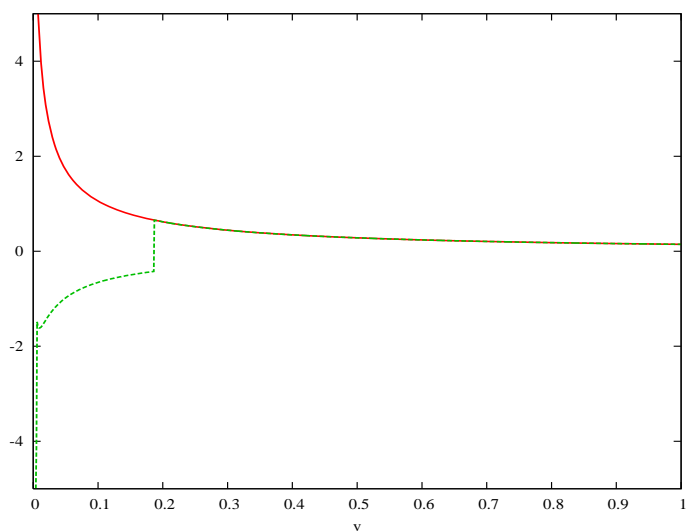

(B)

Figure 1: Complex discontinuities in the Heston model Parameters: $\kappa=6.21, \omega=0.61, \rho=-0.7, \theta=0.019, \mathrm{v}(0)=0.010201, \mathrm{~F}=\mathrm{K}=1, \tau=10$

(A) The principal argument/phase of $\psi_{1}$ with (red solid line) and without (green dashed line) correction (B) Integrand in equation (1) in formulation 1 (green dashed line) vs. formulation 2 (red solid line)

the discontinuities we would get in the argument or phase of $\psi_{1}(\mathrm{v}-\mathrm{i}(\alpha+1))$ if we would use the principal branch only, compared with the argument obtained by applying the rotation count algorithm. In the right panel the impact of these discontinuities on the integrand in equation (1) are shown. Here we used the optimal $\alpha$ and transformed the integrand to the unit interval with a logarithmic transformation, as outlined in Lord and Kahl [2006]. The chosen example is a 10 year at-the-forward European call, whose true price is 0.5266 . The option price found from the green dashed line would have been 0.0373 , a marked difference that will certainly not go unnoticed. At 
smaller maturities however, the differences may not stand out so clearly. With a maturity of 2.5 years there is only one discontinuity; the true option price is 0.2565 whereas the option price found by bluntly using the principal argument in formulation 1 yields 0.2636 . It must be noted that for the parameter set of Duffie et al. the differences are amplified by the large value of $\kappa$. As nowadays values of $\kappa$ are typically smaller, it could well be that the errors have not been detected yet. As the volume of long-dated equity options grows rapidly, this seems just a matter of time.

\subsection{Moment stability}

Before turning to the proof of correctness of the rotation count algorithm and the proof that formulation 2 can be used in conjunction with the principal argument, we make a detour and investigate the issue of moment stability in the Heston model. As we will see in section 4, this issue is intimately linked to the proof that the principal branch can be used in formulation 2 . Though Andersen and Piterbarg [2004] deal with the main issues here, our exposure is different.

Analysing moment stability, or the strip of regularity of the characteristic function, entails analysing the range of $\zeta \in \mathbb{R}$ for which $\phi\left(-\zeta_{\mathrm{i}}\right)<\infty$. Clearly this range will be of the form $\left(\zeta_{-}, \zeta_{+}\right)$. Let $\Lambda_{\mathrm{x}}=\left\{\mathrm{u} \in \mathbb{C} \mid-\operatorname{Im}(\mathrm{u}) \in\left(\zeta_{-}, \zeta_{+}\right)\right\}$, then the absolute value $|\phi(\mathrm{u})|<\infty$ provided that $\mathrm{u} \in \Lambda_{\mathrm{x}}$. The reason for this is that if $\mathrm{u} \in \Lambda_{\mathrm{x}}$, we immediately have:

$$
|\phi(\mathrm{u})|=\left|\mathbb{E}\left[\mathrm{e}^{\mathrm{iu} \ln \mathrm{S}(\mathrm{T})}\right]\right| \leq \mathbb{E}\left[\left|\mathrm{e}^{\mathrm{iu} \ln \mathrm{S}(\mathrm{T})}\right|\right]=\phi(-\operatorname{Im}(\mathrm{u}) \mathrm{i})<\infty
$$

Analysing the moment stability thus boils down to analysing the stability of the system of ODEs in equation (6) for $u=-\zeta i$. As the function A will simply be an integral over $B_{v}$, the stability of $B_{v}$ is what matters. The solution in (8) is cleary stable if and only if the denominator is not equal to zero, modulo those cases where both the numerator and the denominator are zero and (8) remains well-defined. The following theorem gives a necessary and sufficient condition for stability of the solution in (9), and hence also for the characteristic function itself.

\section{Theorem 1}

The coefficient of the variance $\mathrm{B}_{\mathrm{v}}(\mathrm{u}, \tau)$, and hence the full characteristic function, is analytic for $\mathrm{u} \in \Lambda \subset \mathbb{C}$, where $\mathrm{u} \notin \Lambda$ if $\zeta=-\operatorname{Im}(\mathrm{u})$ satisfies one of the two following conditions:

1. $\mathrm{G}(-\zeta \mathrm{i}) \mathrm{e}^{-\mathrm{D}(-\zeta \mathrm{i}) \tau}=1 \wedge \mathrm{D}(-\zeta \mathrm{i}) \neq 0$

2. $\mathrm{D}(-\zeta \mathrm{i})=0 \wedge \tau=-2 \beta(-\zeta \mathrm{i})^{-1}$

\section{Proof:}

First note that it is sufficient to focus on the case where $u=-\zeta i$ with $\zeta \in \mathbb{R}$ due to (14). Let us first check those cases when the numerator of (9) equals zero, namely $\beta=\mathrm{D}$ or $\mathrm{D}=0$. In the first case, the denominator will be equal to 1 and we have $B_{v}(u, \tau)=0$. In the last case we need one application of l'Hôpital's rule to find:

$$
\lim _{D(u) \rightarrow 0} B_{v}(u, \tau)=\lim _{D \rightarrow 0} \frac{e^{-D \tau} \tau(\beta-D)-\left(1-e^{-D \tau}\right)}{\omega^{2} e^{-D \tau \tau} \frac{2 \beta+\beta^{2} \tau-\tau D^{2}}{(\beta+D)^{2}}}=\frac{\beta^{2} \tau}{2 \gamma(2+\beta \tau)}
$$

so that (9) remains well-defined, provided that $\tau \neq-2 \beta(\mathrm{u})^{-1}$ whenever $\mathrm{D}(\mathrm{u})=0$. Note that this can only happen when $\operatorname{Re}(\mathrm{u})=0$, the situation we have here. Combined with the condition following from the denominator of (9) we arrive at the conditions (15)-(16). Note that $\Lambda_{\mathrm{x}} \subset \Lambda$. 


\section{Remark 1}

Given a fixed time to maturity $\tau$, a complex number $\mathrm{u} \notin \Lambda_{\mathrm{x}}$ if:

1. $\mathrm{G}(-\zeta \mathrm{i}) \mathrm{e}^{-\mathrm{D}(-\zeta \mathrm{i}) \tau}=1 \wedge \mathrm{D}(-\zeta \mathrm{i}) \neq 0$

2. $\mathrm{D}(-\zeta \mathrm{i})=0 \wedge \tau \geq-2 \beta(-\zeta \mathrm{i})^{-1}>0$

The only point where the conditions differ with theorem 1 is the second condition. Though the characteristic function may be analytic for values of $\tau>-2 \beta\left(-\zeta_{\mathrm{i}}\right)>0$ whenever $\mathrm{D}\left(-\zeta_{\mathrm{i}}\right)=0$, the $\zeta^{\text {th }}$ moment will be infinite.

The following remark demonstrates that conditions (15) and (18) are sufficient for $\rho \leq \kappa / \omega$.

\section{Remark 2}

Condition (15) is sufficient when $\rho \leq \kappa / \omega$. To show this, let us first analyse $D(-\zeta \mathrm{i})^{2}$, which is a second order polynomial in $\zeta$ :

$$
\begin{aligned}
\mathrm{D}(-\zeta \mathrm{i})^{2} & =\beta(-\zeta \mathrm{i})^{2}-\omega^{2} \zeta(\zeta-1)=(\kappa-\omega \rho \zeta)^{2}-\omega^{2} \zeta(\zeta-1) \\
& =\kappa^{2}+\omega(\omega-2 \kappa \rho) \zeta-\omega^{2}\left(1-\rho^{2}\right) \zeta^{2}
\end{aligned}
$$

with roots equal to:

$$
\zeta_{\mathrm{D} \pm}=\frac{\omega(\omega-2 \kappa \rho) \pm \sqrt{\omega^{2}(\omega-2 \kappa \rho)^{2}+4 \omega^{2}\left(1-\rho^{2}\right) \kappa^{2}}}{2 \omega^{2}\left(1-\rho^{2}\right)}
$$

Note that $\zeta_{\mathrm{D}^{+}} \geq 1$ as $\mathrm{D}(-\mathrm{i})^{2}=(\kappa-\omega \rho)^{2} \geq 0$, and also $\zeta_{\mathrm{D}_{-}}<0$ as $\mathrm{D}(0)^{2}=\kappa^{2}$. The solutions in (21) remain well-defined for values of $\rho \leq \kappa / \omega+\omega / 4 \kappa$. Ensuring that (16) can never occur now comes down to checking that $\tau \neq-2 \beta\left(-\zeta_{\mathrm{D} \pm \mathrm{i}}\right)^{-1}$. It is sufficient to check that $\beta\left(-\zeta_{\mathrm{D} \pm \mathrm{i}} \mathrm{i} \geq 0\right.$. For $\rho$ equal to zero it can immediately be seen that this is true. For $\rho \neq 0$ tedious algebra shows that this inequality is equivalent to:

$$
\operatorname{sgn}(\rho) \cdot \pm \sqrt{\omega^{2}(\omega-2 \kappa \rho)^{2}+4 \omega^{2}\left(1-\rho^{2}\right) \kappa^{2}} \leq \omega|\rho|^{-1}(2 \kappa-\omega \rho)
$$

Analysing all possible cases, we conclude that (16) can never occur for $\zeta=\zeta_{\mathrm{D}-}$, whereas for the positive root of (20), $\zeta=\zeta_{\mathrm{D}^{+}}$, it cannot occur provided that $\rho \leq \kappa / \omega$.

We note that condition (15) together with the restriction that $\rho \leq \kappa / \omega$ also appears in Lee [2004, Appendix A.2], however without any hint as to how it can be derived. To interpret the condition $\rho \leq \kappa / \omega$ we should mention a few words about the use of the correlation parameter in stochastic volatility models. The only fundamental difference between the use of the stochastic volatility driver among various asset classes is the correlation parameter $\rho$. Whereas in an equity or FX context $\rho$ is used to fit to the skew or smile present in that market, the correlation parameter $\rho$ is set to zero when used in a term structure context. Though there is empirical evidence for this, see references in e.g. Andersen and Brotherton-Ratcliffe [2005], the main reason is of a practical nature. If $\rho$ would be unequal to zero, a change of probability measure would change the structure of the stochastic volatility driver and in a term structure context would cause forward rates to appear in its drift. By setting $\rho$ equal to zero this is avoided. In a term structure context therefore 
a displacement coefficient is often added to the underlying in order to be able to fit to the skew. In an equity context where there typically is a skew, we usually find $-1<\rho<-0.7$, see e.g. Jäckel [2004]. Even if a positive value of $\rho$ would be required, one typically finds $\kappa>\omega$ in an implied calibration so that the condition is not in the least bit restrictive. For evidence of this in the literature, see e.g. the parameters of Duffie et al. we used in figure 1, or the parameters found by Bakshi, Cao and Chen [1997] and Schoutens et al. [2004].

Finally, we note that solving $\zeta_{+}$and $\zeta_{-}$from (15) is not a well-posed problem, as there are an infinite number of solutions. To this end it is convenient to use the explosion or critical time analysed in Andersen and Piterbarg [2004]. In Lord and Kahl [2006] we provide good starting solutions for this numerical problem.

\section{Why the rotation count algorithm works}

In Kahl and Jäckel [2005a] the rotation count algorithm was devised, an algorithm that automatically adapts the branch of the complex logarithm such that the characteristic function in formulation 1 is continuous, as it should be. For $\mathrm{a}, \mathrm{b} \in \mathbb{C}$ and $\mathrm{c} \in \mathbb{R}$ the algorithm is basically concerned with the evaluation of $d$ in:

$$
\mathrm{d}=\mathrm{ae}^{\mathrm{b}}+\mathrm{c}
$$

such that its argument is kept continuous if both the complex function b and the argument of a are continuous functions. In the algorithm we will use both the classical and polar representations of a complex number, i.e. for $\mathrm{z} \in \mathbb{C}$ we will write $\mathrm{z}=\mathrm{z}_{\mathrm{r}}+\mathrm{z}_{\mathrm{i}} \mathrm{i}$ with $\mathrm{z}_{\mathrm{r}}=\operatorname{Re}(\mathrm{z})$ and $\mathrm{z}_{\mathrm{i}}=\operatorname{Im}(\mathrm{z})$, as well as $z=|z| e^{i z_{\theta}}$ with $|z|$ being its radius and $z_{\theta}$ its argument ${ }^{5}$. The algorithm follows as:

$$
\begin{aligned}
& \text { 1. Calculate the phase interval of } a^{b} \text { as } n=\left[\frac{1}{2 \pi}\left(a_{\theta}+b_{i}+\pi\right)\right] \text {, equal to } 0 \text { if it is in }[-\pi, \pi) \text {; } \\
& \text { 2. }|d|=\left|a e^{b}+c\right| \\
& \text { 3. } d_{\theta}=\arg \left(a e^{b}+c\right)+2 \pi \text { n where arg denotes the principal argument. }
\end{aligned}
$$

Algorithm 1: The rotation count algorithm of Kahl and Jäckel

The premise under which algorithm 1 is valid is that the addition of the real number c does not change the phase interval of the resulting complex number. If this is indeed the case, we can use two successive applications of the rotation count algorithm to evaluate the ratio $\psi_{1}$ in formulation 1 , see equation (12). First we evaluate $d_{1}(u, \tau)=c(u) e^{D(u) \tau}-1$, subsequently $d_{2}(u)=c(u)-1$, and finally we write:

$$
\psi_{1}(\mathrm{u}, \tau)=\frac{\left|\mathrm{d}_{1}(\mathrm{u}, \tau)\right|}{\left|\mathrm{d}_{2}(\mathrm{u})\right|} \mathrm{e}^{\mathrm{i}\left(\mathrm{d}_{1 \theta}(\mathrm{u}, \tau)-\mathrm{d}_{2 \theta}(\mathrm{u})\right)}
$$

The following lemma shows that evaluating $\psi_{1}$ in this way leads to a continuous characteristic function, if the premise of the rotation count algorithm is valid.

\section{Lemma 1}

Assume that the premise of the rotation count algorithm is valid and that $\mathrm{u}: \mathbb{R} \rightarrow \Lambda_{\mathrm{x}}$ describes a continuous path in the complex plane. In this case, applying the rotation count algorithm to

\footnotetext{
${ }^{5}$ Note that this is not necessarily the principal argument.
} 
evaluate the argument of $\psi_{1}(\mathrm{u}(\mathrm{x}), \tau)$ as $\mathrm{d}_{1 \theta}(\mathrm{u}(\mathrm{x}), \tau)-\mathrm{d}_{2 \theta}(\mathrm{u}(\mathrm{x}))$ yields a continuous characteristic function.

\section{Proof:}

Though the arguments $d_{1 \theta}$ and $d_{2 \theta}$ are not necessarily continuous, any discontinuities caused by $\arg (\mathrm{c})$ are cancelled out as they appear in both terms. The remaining term that could cause a discontinuity is $\mathrm{D}(\mathrm{u})$. For $-\operatorname{Im}(\mathrm{u}) \notin\left[\zeta_{\mathrm{D}^{-}}, \zeta_{\mathrm{D}^{+}}\right]$the function $\mathrm{D}(\mathrm{u})$ is continuous, unless $\mathrm{u}(\mathrm{x})$ crosses the imaginary axis, as for $-\mathrm{y} \notin\left[\zeta_{\mathrm{D}-}, \zeta_{\mathrm{D}+}\right]$ we have $\lim _{\mathrm{x} \uparrow 0} \mathrm{D}(\mathrm{x}+\mathrm{yi})=\varlimsup_{\mathrm{x} \downarrow 0} \mathrm{D}(\mathrm{x}+\mathrm{yi})$. Nevertheless this is not a problem either, as for $\mathrm{u} \in \Lambda_{\mathrm{x}}$ the characteristic function is real on the imaginary axis, so that this discontinuity in $\mathrm{D}(\mathrm{u})$ does not have an impact on the full characteristic function. Under the premise of the rotation count algorithm the assertion is thus true.

The remaining problem is now to check that the premise of the rotation count algorithm is true. Consider the following complex function:

$$
f(x)=(1-x)-\left(\frac{1}{2}-x\right) i
$$

where $x \in \mathbb{R}$. In the following figure we have drawn $f(x)+1, f(x), f(x)-1$ and $f(x)-2$ in the complex plane, as well as their principal arguments:

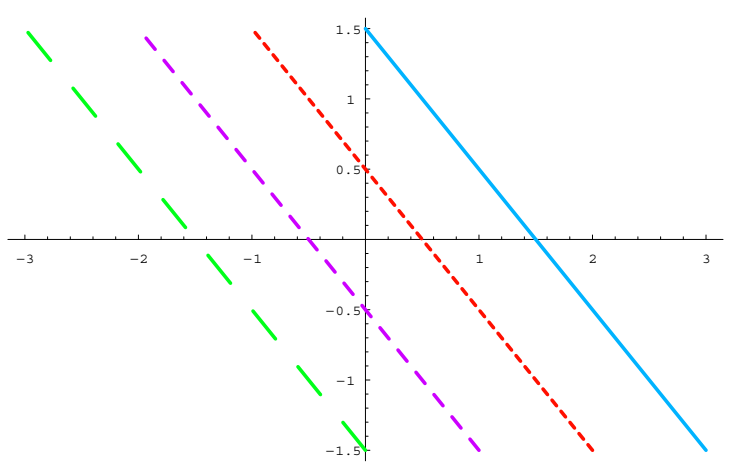

(A)

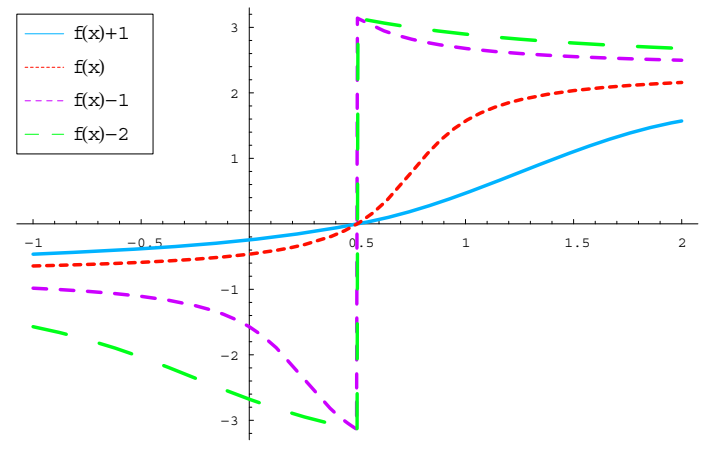

(B)

Figure 2: Addition of a real number to a complex number can change the phase interval

(A) $f(x)+1, f(x), f(x)-1$ and $f(x)-2$ in the complex plane

(B) Argument of all four functions as a function of $\mathrm{x}$

While $f(x)+1$ and $f(x)$ do have a continuous principal argument, $f(x)-1$ and $f(x)-2$ clearly do not. The discontinuities here are caused by the fact that these functions make a transition from the second to the third quadrant, implying that the principal argument changes from the interval $[1 / 2 \pi, \pi)$ to $[-\pi,-1 / 2 \pi)$, clearly causing a jump. The following lemma formalises this observation.

\section{Lemma 2}

Consider a continuous function $\mathrm{z}: \mathbb{R} \rightarrow \mathbb{C}$, where both the real and complex part of $\mathrm{z}$ are strictly monotone. Assume that $\mathrm{z}$ never passes through the origin ${ }^{6}$. Adding $\mathrm{y} \in \mathbb{R}$ where $\mathrm{y} \neq 0$ to $\mathrm{z}(\mathrm{x})$

\footnotetext{
${ }^{6}$ We avoid trajectories that pass through the origin as the principal argument of zero is undefined.
} 
does not add any discontinuities to the principal argument of $\mathrm{z}(\mathrm{x})+\mathrm{y}$ when compared to the principal argument of $\mathrm{z}(\mathrm{x})$, if and only if:

- $\operatorname{Re}(\mathrm{z}(\mathrm{x})) \notin(-\mathrm{y}, 0)$ for $\mathrm{y}>0$ whenever $\operatorname{Im}(\mathrm{z}(\mathrm{x}))$ changes sign;

- $\operatorname{Re}(\mathrm{z}(\mathrm{x})) \notin(0,-\mathrm{y})$ for $\mathrm{y}<0$ whenever $\operatorname{Im}(\mathrm{z}(\mathrm{x}))$ changes sign.

Proof: See the appendix.

Lemma 2 immediately gives necessary and sufficient conditions under which algorithm 1 will work. For the rotation count algorithm to work when applied to the evaluation of $\psi_{1}$ in (24), it is hence sufficient to ensure that whenever the imaginary parts of $c(u)$ or $c(u) e^{\mathrm{D}(u) \tau}$ are zero, their real parts are not in the interval $[0,1]$. In this respect the following lemma is very useful.

\section{Lemma 3}

If $\rho \leq \kappa / \omega$, or $\operatorname{Im}(\mathrm{u}) \geq \mathrm{y}_{2}$ and $\kappa / \omega \leq \rho \leq 2 \kappa / \omega$, we have:

$$
|G(u)|=\left|\frac{\beta(u)-D(u)}{\beta(u)+D(u)}\right| \leq 1
$$

where the inequality is strict, except for some cases where $\operatorname{Re}(u)=0$ or $\rho=2 \kappa / \omega$.

Proof: See the appendix.

We only require one additional lemma before we can prove the main theorem of this section.

\section{Lemma 4}

For $\mathrm{u} \in \Lambda_{\mathrm{x}}$ we can only have $\mathrm{ce}^{\mathrm{D} \tau}=1$ or $\mathrm{c}=1$ when $\mathrm{D}=0$. In this case $\psi_{1}$ and $\psi_{2}$ will never cross the negative real line.

\section{Proof:}

Again, we write $u=x+y i$ for $x, y \in \mathbb{R}$. It is easy to check that $c$ can only equal 1 when $D=0$. From case 1 of the proof of lemma 3 in the appendix we know that this can only be the case when $\mathrm{x}=0$. Note that we have:

$$
\lim _{\mathrm{D}(\mathrm{u}) \rightarrow 0} \psi_{1}(\mathrm{u}, \tau)=\lim _{\mathrm{D}(\mathrm{u}) \rightarrow 0} \psi_{2}(\mathrm{u}, \tau)=1+\frac{1}{2} \beta(\mathrm{u}) \tau
$$

Since $\mathrm{u} \in \Lambda_{\mathrm{x}}$ we can only have $\mathrm{D}(\mathrm{yi})=0$ when $\tau<\max \left(-2 \beta(\mathrm{yi})^{-1}, 0\right)$. The condition on $\tau$ precludes $\psi_{1}$ and $\psi_{2}$ from crossing the negative real line.

Lemmas 3 and 4 have now given us enough machinery to be able to prove the main theorem of this section, which provides sufficient conditions under which the rotation count algorithm is guaranteed to work when applied to the Heston model.

\section{Theorem 2}

The rotation count algorithm can safely be applied to the Heston model as long as the characteristic function is evaluated in $\mathrm{u} \in \Lambda_{\mathrm{x}}$ and the parameters are such that either $\rho \leq \kappa / \omega$, or $\operatorname{Im}(\mathrm{u}) \geq-\kappa /(\rho \omega)$ and $\kappa / \omega \leq \rho<2 \kappa / \omega$. Care has to be taken only when $\operatorname{Re}(\mathrm{u})=0$ and $\mathrm{D}(\mathrm{u})=0$. In this case $\psi_{1}$ should be directly evaluated as in (27). 


\section{Proof:}

Remember that $c=1 / G$, so that for these parameter combinations we have $|c| \geq 1$ by virtue of lemma 3. Furthermore:

$$
\left|c e^{D \tau}\right|=|c| e^{D_{r} \tau} \geq|c| \geq 1
$$

since we use the convention that the real part of the square root is nonnegative and $\tau \geq 0$. If the inequality is strict, it implies that whenever the imaginary parts of $c(u)$ or $c(u) e^{D(u) \tau}$ are zero their real parts are not in the interval [0,1]. Since we have excluded the case that $\rho=2 \kappa / \omega$ here, we need only worry about cases where $\operatorname{Re}(\mathrm{u})=0$ and the inequality in (26) turns into an equality. If we write $u=y i$ for $y \in \mathbb{R}$, the condition $|G|=1$ is equivalent to:

$$
\operatorname{Re}(\beta(y i)) \cdot \operatorname{Re}(D(y i))=0
$$

The only possibilities for problems are therefore $\beta=0$ (as its imaginary part is always zero here) or $D_{r}=0$. When $\beta=0, G=-1$ so that there is no problem. When $D_{r}=0, G$ lies on the unit circle. This implies that $\left|\mathrm{ce}^{\mathrm{D \tau}}\right|=|\mathrm{c}|=1$. The only possible problem could therefore be that either of the two complex numbers equal 1 . Lemma 4 shows that in this case there is no problem either, as long as we evaluate $\psi_{1}$ as in (27).

\section{Remark 3}

As in section 2, we mention that the parameter restrictions under which the rotation count algorithm is guaranteed to work are not restrictive at all, as the most important cases of highly negative correlation and zero correlation are covered. As in an implied calibration one typically finds the speed of mean reversion $\kappa$ to be larger than or equal to the volatility of variance $\omega$, the restriction that $\rho \leq \kappa / \omega$ will typically be satisfied. Even if $\kappa / \omega<\rho<2 \kappa / \omega$ there is still a wide range of complex numbers $u$ in which we can evaluate the characteristic function in conjunction with the rotation count algorithm. For the option pricing application outlined in the introduction, the restrictions placed on $\operatorname{Im}(\mathrm{u})$ imply that values of $\alpha \leq-1 / 2$ can certainly be used.

\section{Why the principal branch can be used}

Though theorem 2 from the previous section has taught us that the rotation count algorithm of Kahl and Jäckel will indeed work when applied to formulation 1, we already remarked earlier that using formulation 2 in conjunction with the principal branch of the complex logarithm does not seem to cause any discontinuities in the characteristic function. A distinct advantage of formulation 2 is its numerical stability. Note that $D(x+y i)$ tends to $\omega \sqrt{1-\rho^{2}} x$ as $x$ tends to infinity, so that the real part of $\mathrm{D}(\mathrm{u})$ can become quite large when calculating option prices. While this leads to numerical instabilities in the calculation of $\psi_{1}$ (formulation 1), as mentioned in Kahl and Jäckel's paper, $\psi_{2}$ (formulation 2) is much better behaved. If we can therefore rigorously prove that formulation 2 does not cause any complex discontinuities, it should be preferred due to its much easier implementation and numerical stability. The following theorem provides exactly this result. Keep in mind that if we restrict the complex logarithm to its principal branch, a complex continuous function that does not pass through the origin will remain continuous when its logarithm is taken, if and only if the original function does not cross the negative real line. 


\section{Theorem 3}

Let $\mathrm{u} \in \Lambda_{\mathrm{x}}, \rho \leq \kappa / \omega$, or $\operatorname{Im}(\mathrm{u}) \geq-\kappa /(\rho \omega)$ and $\kappa / \omega \leq \rho<2 \kappa / \omega$. If we are evaluating the characteristic function by means of formulation 2 in (11), the principal branch of the complex logarithm is the right one. When $\operatorname{Re}(\mathrm{u})=0$ and $\mathrm{D}(\mathrm{u})=0$ we have to evaluate $\psi_{2}$ as in (27).

\section{Proof:}

We have to prove that $\psi_{2}(\mathrm{u}, \tau)$, defined in (11), never crosses the negative real line for the parameter combinations under consideration. Suppose that it does, i.e. that:

$$
\psi_{2}(\mathrm{u}, \tau)=\frac{\mathrm{G}(\mathrm{u}) \mathrm{e}^{-\mathrm{D}(\mathrm{u}) \tau}-1}{\mathrm{G}(\mathrm{u})-1}=-\xi
$$

for $\xi \geq 0$. Lemma 4 handles the cases where the numerator or the denominator equal zero, and shows that in this case $\psi_{2}$ cannot cross the negative real line. Therefore we can assume that $\mathrm{G} \neq 1$ and that $\xi$ must be strictly larger than zero. We can then rearrange (30) as:

$$
\mathrm{G}\left(\xi+\mathrm{e}^{-\mathrm{D} \tau}\right)=\xi+1
$$

In view of $\xi, \tau \geq 0,|G| \leq 1$ and $D_{r} \geq 0$ we can take the modulus of the left-hand side and show:

$$
\left|\mathrm{G}\left(\xi+\mathrm{e}^{-\mathrm{D} \tau}\right)\right| \leq|\mathrm{G}| \cdot\left(\xi+\mathrm{e}^{-\mathrm{D}_{\mathrm{r}} \tau}\right) \leq \xi+1
$$

where the first inequality is strict unless $D_{\mathrm{i}} \tau=2 n \pi$ with $n \in \mathbb{Z}$, and the second inequality is strict unless $|G|=1$ and $D_{r}=0$. The equality in (31) can therefore only occur if $|G|=1, D_{r}=0$ and $D_{i} \tau=2 n \pi$. From the proof of theorem 2 we know that $|G|=1$ and $D_{r}=0$ can only occur when $\operatorname{Re}(\mathrm{u})=0$, so that we can write $\mathrm{u}=$ yi. We can assume that $\mathrm{D}_{\mathrm{i}} \neq 0$ as $\mathrm{D}=0$ implies $\mathrm{G}=1$. Plugging all these values into $\psi_{2}$ shows that its imaginary value can only be zero when $\beta_{\mathrm{r}}=0$ as well, and since $\beta_{\mathrm{i}}=0$ for $\mathrm{u}=\mathrm{yi}$, this implies $\beta=0$. Finally, note that for $\mathrm{u}=\mathrm{yi}, \mathrm{D}_{\mathrm{r}}=0, \mathrm{D}_{\mathrm{i}} \neq 0$ and $\beta=0$ we have:

$$
\psi_{2}(\mathrm{yi}, \tau)=\frac{1}{2}\left(1+\mathrm{e}^{-\mathrm{D}_{\mathrm{i}}(\mathrm{yi}) \tau}\right) \geq \frac{1}{2}
$$

which concludes the proof.

The proof of theorem 3 is reminiscent of the proof of theorem 2, as both proofs hinge on the result of lemma 3 , the result that for most parameter combinations $|G| \leq 1$. In that sense using the rotation count algorithm on formulation 1, or directly using formulation 2 and restricting the complex logarithm to its principal branch, are equivalent approaches. As formulation 2 is much easier to implement and numerically more stable, it should be the preferred formulation.

The line of reasoning used to prove theorems 2 and 3 unfortunately does not lead to a universal proof. Nonetheless extensive experiments outside the range of parameters indicated by theorems 2 and 3 have as yet not resulted in a single counterexample. We therefore end this section with an unproven conjecture that formulation 2, or indeed applying the rotation count algorithm to formulation 1, leads to a continuous characteristic function. We suspect its proof is very intimately related to the subject of moment stability. 


\section{Conjecture}

Evaluating the Heston characteristic function by means of formulation 2, where we restrict the complex logarithm to its principal branch, or by means of formulation 1, to which we then apply the rotation count algorithm, ensures that the characteristic function of the Heston model remains continuous.

\section{Related issues in other models}

Having analysed the Heston characteristic function in great detail, it is time to turn to other models and see what can be done about the complex discontinuities in their characteristic functions. The characteristic functions we analyse stem from the Variance Gamma model, the Schöbel-Zhu model, the exact simulation algorithm of the Heston model and finally Matytsin's extension of the Heston model which allows for jumps in both the asset and the variance.

\subsection{The Variance Gamma model}

To demonstrate that other models besides stochastic volatility models may have complex discontinuities, we turn to a model of the exponential Lévy class, the Variance Gamma (VG) model, first introduced by Madan and Seneta [1990]. Here the underlying asset is modelled as:

$$
\mathrm{S}(\mathrm{t})=\mathrm{F}(\mathrm{t}) \exp (\omega \mathrm{t}+\theta \mathrm{G}(\mathrm{t})+\sigma \mathrm{W}(\mathrm{G}(\mathrm{t})))
$$

where $\mathrm{W}(\mathrm{t})$ is a standard Brownian motion, $\mathrm{G}(\mathrm{t})$ is a Gamma process with parameter $v$ and $\mathrm{F}(\mathrm{t})$ is the forward price of the underlying stock at time t. Without loss of generality we assume that both $\sigma$ and $v$ are strictly positive. The parameter $\omega$ is chosen such that the exponential term in (34) has an expectation equal to 1:

$$
\omega=\frac{1}{v} \ln \left(1-\theta v-\frac{1}{2} \sigma^{2} v\right)
$$

To simplify notation we introduce $f(t)=\ln F(t)$ and $\tilde{f}(t)=f(t)+\omega t$. If we denote $\tau$ as the time to maturity, the conditional characteristic function of the VG model is then specified as:

$$
\phi(u)=\mathbb{E}\left[e^{i u \ln s(T)}\right]=\frac{\exp (i u \tilde{f}(T))}{\left(1-i u\left(\theta+\frac{1}{2} i \sigma^{2} u\right) v\right)^{\tau / v}}
$$

The $\zeta^{\text {th }}$ moment of the underlying asset exists as long as $\zeta \in\left(\zeta_{-}, \zeta_{+}\right)$, defined by:

$$
\zeta_{ \pm}=-\frac{\theta}{\sigma^{2}} \pm \sqrt{\frac{\theta^{2}}{\sigma^{4}}+\frac{2}{v \sigma^{2}}}
$$

so that the extended characteristic function in (36) is well-defined for $\mathrm{u} \in \Lambda_{\mathrm{x}}$, which is as before defined by $\left\{u \in \mathbb{C} \mid-\operatorname{Im}(\mathrm{u}) \in\left(\zeta_{-}, \zeta_{+}\right)\right\}$. As the VG model is fully time-homogeneous, the maximum and minimum allowed moments do not depend on the maturity $\mathrm{T}$, in contrast with what we had in the Heston model. In the denominator of (36) we are using the complex power function, again a multivalued function. The complex discontinuities in the Heston model were in fact also caused by the branch switching of the complex power function. Although the 
characteristic exponent of the Heston model contains a complex logarithm, this term is multiplied by $-2 \kappa \theta / \omega^{2}$ and subsequently its exponent is taken. In essence we are thus raising $\psi_{1}$ or $\psi_{2}$ (depending on which formulation we use) to the power of $-2 \kappa \theta / \omega^{2}$, so that the branch switching of the complex power function is the cause of our complex discontinuities. As mentioned in section 2.2, if $2 \kappa \theta / \omega^{2} \in \mathbb{N}$, there will be no discontinuities if we restrict the logarithm to its principal branch.

If we here restrict the complex power function to its principal branch, i.e. if we evaluate $\mathrm{z}^{\alpha}$ for $\mathrm{z} \in \mathbb{C}$ and $\alpha \in \mathbb{R}$ as $|z|^{\alpha} \mathrm{e}^{\mathrm{i} \alpha \arg (\mathrm{z})}$, with $\arg (\mathrm{z})$ being the principal argument of $\mathrm{z}$, then the characteristic function of the VG model in (36) will only be continuous if $1-i u\left(\theta+\frac{1}{2} i \sigma^{2} u\right) v$ does not cross the negative real line. In the following theorem it is proven that this never occurs.

\section{Theorem 4}

When evaluating the characteristic function of the VG model in $\mathrm{u} \in \Lambda_{\mathrm{x}}$, we can safely restrict the complex power function to its principal branch.

\section{Proof:}

If we write $u=x+y i$, then the imaginary part of $1-i u\left(\theta+\frac{1}{2} i \sigma^{2} u\right) v$ can only equal zero when either $\mathrm{x}=0$ or $\mathrm{y}=\theta / \sigma^{2}$. For $\mathrm{x}=0$ we have:

$$
1-\mathrm{iu}\left(\theta+\frac{1}{2} \mathrm{i} \sigma^{2} \mathrm{u}\right) v=1+v \theta y-\frac{1}{2} v \sigma^{2} \mathrm{y}^{2}
$$

which for $\mathrm{u} \in \Lambda_{\mathrm{x}}$, or here $-\mathrm{y} \in\left(\zeta_{-}, \zeta_{+}\right)$, is strictly positive. For $\mathrm{y}=\theta / \sigma^{2}$ we find:

$$
1-i u\left(\theta+\frac{1}{2} i \sigma^{2} u\right) v=1+\frac{v \theta^{2}}{2 \sigma^{2}}+\frac{1}{2} v \sigma^{2} x^{2} \geq 1
$$

so that $1-i u\left(\theta+\frac{1}{2} i \sigma^{2} u\right) v$ can clearly never cross the negative real line. The principal branch of the complex power function is the correct one, as this is the only one that leads to real values for the moment generating function.

\subsection{The Schöbel-Zhu model}

The first mention of discontinuities in characteristic functions caused by the branch switching of the complex logarithm or indeed the complex power function was in the article of Schöbel and Zhu [1999], who encountered these problems when implementing their extension of the Stein and Stein model to allow for non-zero correlation between the underlying asset and the stochastic volatility process. We will investigate whether, as in the Heston model, there is a formulation of the characteristic function that for all relevant parameter values will not lead to any discontinuities. Under the risk-neutral pricing measure the underlying asset in the Schöbel-Zhu model evolves according to the following set of SDEs:

$$
\begin{aligned}
& \mathrm{dS}(\mathrm{t})=\mu(\mathrm{t}) \mathrm{S}(\mathrm{t}) \mathrm{dt}+\sigma(\mathrm{t}) \mathrm{S}(\mathrm{t}) \mathrm{dW}_{\mathrm{S}}(\mathrm{t}) \\
& \mathrm{d} \sigma(\mathrm{t})=-\kappa(\sigma(\mathrm{t})-\theta) d \mathrm{dt}+\omega \mathrm{dW}_{\sigma}(\mathrm{t})
\end{aligned}
$$

where the Brownian motions satisfy $\mathrm{dW}_{\mathrm{s}}(\mathrm{t}) \cdot \mathrm{dW}_{\sigma}(\mathrm{t})=\rho \mathrm{dt}$. The difference with the Heston model is that instead of the stochastic variance, the stochastic volatility itself here follows an Ornstein- 
Uhlenbeck process. A problem with the Schöbel-Zhu model, as noted by e.g. Jäckel [2004], is that when the volatility process becomes negative, the sign of the instantaneous correlation between $S$ and $\sigma$ effectively changes. This is economically implausible.

Starting from the Heston model, a well-educated guess to recognise an affine model in (40) would be to consider $\ln S$ and $\sigma$. It turns out that this is not an affine model, as the variance $\sigma^{2}$ enters the drift of $\ln \mathrm{S}$. For this reason the Schöbel-Zhu model is often referred to as being a nonaffine model. It turns out that by using the classification of Gaspar [2004] and Cheng and Scaillet [2005] we can conclude that it is a linear-quadratic model, and by the latter paper therefore equivalent to an affine model, provided we use the right coordinates. The only state variable we have to add is the variance itself, $v(t)=\sigma(t)^{2}$ :

$$
d v(t)=2 \sigma(t) d \sigma(t)+\omega^{2} d t=\left(-2 \kappa v(t)+2 \kappa \theta \sigma(t)+\omega^{2}\right) d t+2 \omega \sigma(t) d W_{\sigma}(t)
$$

One can check that the model is certainly affine in $\ln \mathrm{S}, \sigma$ and $\mathrm{v}$, and its characteristic function will therefore have the same exponentially affine form as (5):

$$
\phi(u)=\mathbb{E}\left[e^{i u \ln s(T)}\right]=\exp \left(\text { iuf }+A(u, \tau)+B_{\sigma}(u, \tau) \cdot \sigma(0)+B_{v}(u, \tau) \cdot v(0)\right)
$$

where $A, B_{\sigma}$ and $B_{v}$ can be solved from the following system of ODEs:

$$
\begin{aligned}
\frac{d B_{v}}{d \tau} & =\hat{\alpha}(u)-\beta(u) B_{v}+\gamma B_{v}^{2} \\
\frac{d B_{\sigma}}{d \tau} & =2 \kappa \theta B_{v}+\left(-\frac{1}{2} \beta(u)+\gamma B_{v}\right) \cdot B_{\sigma} \\
\frac{d A}{d \tau} & =\kappa \theta B_{\sigma}+\frac{1}{2} \omega^{2} B_{\sigma}^{2}+\omega^{2} B_{v}
\end{aligned}
$$

subject to the initial conditions $\mathrm{B}_{\mathrm{v}}(\mathrm{u}, 0)=\mathrm{B}_{\sigma}(\mathrm{u}, 0)=\mathrm{A}(\mathrm{u}, 0)=0$. The auxiliary variables are similar to the ones defined in the Heston model, namely $\hat{\alpha}(\mathrm{u})=-\frac{1}{2} \mathrm{u}(\mathrm{i}+\mathrm{u}), \beta(\mathrm{u})=2(\kappa-\rho \omega u \mathrm{i})$ and $\gamma=2 \omega^{2}$. Indeed, there are more similarities with the Heston model. Following remarks by both Heston and Schöbel-Zhu, we know that when $\theta=0$, the Schöbel-Zhu model collapses to a particular instance of the Heston model as can be seen from equation (41) - the variance then has a mean-reversion speed of $2 \kappa$, a volatility of variance equal to $2 \omega$ and a mean-reversion level of $\omega^{2} / 2 \kappa$. If we denote the Heston characteristic function as $\phi_{H}(\mathrm{u}, \mathrm{S}(0), \mathrm{v}(0), \kappa, \omega, \theta, \rho, \tau)$, the Schöbel-Zhu characteristic function becomes:

$$
\begin{aligned}
\phi_{\mathrm{sz}}(\mathrm{u}, \mathrm{S}(0), \sigma(0), \kappa, \omega, \theta, \rho, \tau) & =\phi_{\mathrm{H}}\left(\mathrm{u}, \mathrm{S}(0), \sigma(0)^{2}, 2 \kappa, 2 \omega, \omega^{2} / 2 \kappa, \rho, \tau\right) \\
& \cdot \exp \left(\mathrm{A}_{\sigma}(\tau)+\mathrm{B}_{\sigma}(\tau) \cdot \sigma(0)\right)
\end{aligned}
$$

where $A_{\sigma}$ follows the ODE $\frac{d A_{\sigma}}{d \tau}=\kappa \theta B_{\sigma}+\frac{1}{2} \omega^{2} B_{\sigma}^{2}$. By recognising a special case of the Heston model within the Schöbel-Zhu model, it is immediately clear that the discontinuities can be avoided in the same way as in the Heston model - even the conditions on the parameters remain the same, being $\rho \leq \kappa / \omega$ or $\kappa / \omega<\rho<2 \kappa / \omega$ and $\operatorname{Im}(\mathrm{u}) \geq-\kappa /(\rho \omega)$. As in the Heston model, these conditions are not in the least bit restrictive. Note that in Schöbel and Zhu's original paper a 
different formulation of the characteristic function is used: the term under the complex logarithm is different to that found by using (44) in conjunction with either formulation 1 or 2 . For completeness we provide the remainder of the characteristic function here. Tedious though straightforward manipulations show that $\mathrm{A}_{\sigma}$ and $\mathrm{B}_{\sigma}$ can be solved in closed-form as:

$$
\begin{aligned}
\mathrm{B}_{\sigma}(\mathrm{u}, \tau) & =\kappa \theta \frac{\beta-\mathrm{D}}{\mathrm{D} \omega^{2}} \frac{\left(1-\mathrm{e}^{-\frac{1}{2} \mathrm{D} \tau}\right)^{2}}{1-\mathrm{Ge}^{-\mathrm{D} \tau}} \\
\mathrm{A}_{\sigma}(\tau) & =\frac{(\beta-\mathrm{D}) \kappa^{2} \theta^{2}}{2 \mathrm{D}^{3} \omega^{2}}\left(\beta(\mathrm{D} \tau-4)+\mathrm{D}(\mathrm{D} \tau-2)+\frac{4 \mathrm{e}^{-\frac{1}{2} \mathrm{D} \tau}\left(\frac{\mathrm{D}^{2}-2 \beta^{2}}{\beta+\mathrm{D}} \mathrm{e}^{-\frac{1}{2} \mathrm{D} \tau}+2 \beta\right)}{1-\mathrm{Ge}^{-\mathrm{D} \tau}}\right)
\end{aligned}
$$

with D defined as before in the Heston model.

\subsection{The exact simulation algorithm of the Heston model}

Though the Heston model was originally proposed in 1993, an exact simulation algorithm for the SDEs in (3) was not published until recently by Broadie and Kaya [2006]. Though it goes too far to outline the full algorithm in this paper, a crucial step of the algorithm is the simulation of the integrated square root process conditional upon its begin and endpoint. As this distribution is not known in closed-form, Broadie and Kaya chose to simulate from it by inverting its cumulative distribution function, which is itself found by inversion of the characteristic function:

$$
\begin{aligned}
\phi(u)= & \mathbb{E}\left[\exp \left(i u \int_{s}^{t} v(u) d u\right) \mid v(s), v(t)\right]=\frac{D(u) e^{-\frac{1}{2}(D(u)-\kappa) \tau}\left(1-e^{-\kappa \tau}\right)}{\kappa\left(1-e^{-D(u) \tau}\right)} \\
& \cdot \exp \left(\frac{v(s)+v(t)}{\omega^{2}} \cdot\left[\frac{\kappa\left(1+e^{-\kappa \tau}\right)}{1-e^{-\kappa \tau}}-\frac{D(u)\left(1+e^{-D(u) \tau}\right)}{1-e^{-D(u) \tau}}\right]\right) \\
& \cdot \frac{I_{\frac{1}{2} v-1}\left(\sqrt{v(s) v(t)} \cdot \frac{4 D(u) e^{-\frac{1}{2} D(u) \tau}}{\omega^{2}\left(1-e^{-D(u) \tau}\right)}\right)}{I_{\frac{1}{2} v-1}\left(\sqrt{v(s) v(t)} \cdot \frac{4 \mathrm{e}^{-\frac{1}{2} \kappa \tau}}{\omega^{2}\left(1-e^{-\kappa \tau}\right)}\right)}
\end{aligned}
$$

with $\mathrm{D}(\mathrm{u})=\sqrt{\kappa^{2}-2 \omega^{2} \mathrm{iu}}$, the degrees of freedom $v=4 \kappa \theta / \omega^{2}$ and $\mathrm{I}_{v}$ representing the modified Bessel function of the first kind. Finally, $\tau$ equals $t-s$. As the characteristic function in (46) depends non-trivially on the two realisations $v(s)$ and $v(t)$, major parts of the calculations cannot be precomputed, so that this step of the algorithm will be very time-consuming. It is therefore not surprising that Lord, Koekkoek and Van Dijk [2006] find that several biased simulation schemes, among which their own full truncation scheme and the scheme proposed by Kahl and Jäckel [2005b], outperform the exact simulation scheme in terms of both speed and accuracy, even when we only require the asset value at one time instance. Nonetheless, the exact simulation method can be very useful as a benchmark.

The modified Bessel function of the first kind is characterised by the following power series:

$$
I_{v}(z)=\left(\frac{1}{2} z\right)^{v} \sum_{j=0}^{\infty} \frac{\left(\frac{1}{4} z^{2}\right)^{j}}{j ! \Gamma(v+j+1)}
$$


where $\Gamma$ is the gamma function and $\mathrm{z} \in \mathbb{C}$. If we restrict the complex power function used in (47) to its principal branch, the characteristic function in (46) is found to be discontinuous. The key issue is therefore to find a way to keep track of the correct branch of the complex power function. The following theorem achieves just this.

\section{Theorem 5}

Let us first define $f(u)=\frac{D(u) e^{-\frac{1}{2} D(u) \tau}}{1-e^{-D(u) \tau}}$. In order to evaluate $I_{\frac{1}{2} v-1}\left(\frac{4}{\omega^{2}} \sqrt{v(s) v(t)} \cdot f(u)\right)$ in the characteristic function in (46), we have to evaluate $z^{v}$ in $(47)$ as $\exp (v \ln (z))$, where the logarithm is kept continuous if we evaluate it as:

$$
\ln f(u)=g(u)-\operatorname{Im}(g(y i))+\operatorname{Im}(\ln f(y i))
$$

Here we implicitly defined:

$$
g(u)=\ln D(u)-\frac{1}{2} D(u) \tau-\ln \left(1-e^{-D(u) \tau}\right)
$$

where the complex logarithm is restricted to its principal branch. If $\mathrm{D}(\mathrm{u})=0$, which happens only when $\operatorname{Re}(\mathrm{u})=0$ and $\operatorname{Im}(\mathrm{u})=-\kappa^{2} / 2 \omega^{2}$, we have:

$$
\mathrm{g}(\mathrm{u})=-\ln \tau
$$

\section{Proof:}

As $\frac{4}{\omega^{2}} \sqrt{\mathrm{v}(\mathrm{s}) \mathrm{v}(\mathrm{t})}$ is merely a real-valued constant, it is sufficient to focus on $\mathrm{f}(\mathrm{u})$. The challenge here is to keep its argument continuous. We will first prove that $g(u)$ is a continuous function. First of all, D(u) itself can never cross the negative real line, as we here use the convention that the real part of the square root is nonnegative. The only possible problem therefore is $\mathrm{D}(\mathrm{u})=0$ :

$$
\lim _{D \rightarrow 0} \frac{D e^{-\frac{1}{2} \mathrm{D} \tau}}{1-\mathrm{e}^{-\mathrm{D} \tau}}=\frac{1}{\tau}>0
$$

so that $\mathrm{g}(\mathrm{u})$ should be evaluated as in (50) in this case. As for $1-\mathrm{e}^{-\mathrm{D}(\mathrm{u}) \tau}$, we can conclude that this too will never cross the negative real line, unless $D_{r}=0$ and $D_{i} \tau=2 n \pi$ for $n \in \mathbb{Z}$. If we write $\mathrm{u}=\mathrm{x}+\mathrm{yi}$ this can only occur for $\mathrm{x}=0$ and $\kappa^{2}+2 \omega^{2} \mathrm{y}=-(2 \mathrm{n} \pi / \tau)^{2}$. One can check that unless $\mathrm{n}=0$ the quotient is not well-defined, and indeed the moment-generating function will explode. We suspect therefore that $\zeta_{-}=-\infty$ and $\zeta_{+}=\frac{\kappa^{2}+4 \pi^{2} / \tau^{2}}{2 \omega^{2}}$. When $\mathrm{n}=0$ we have $\mathrm{D}=0$ so that (50) applies once again. We conclude that $\mathrm{g}(\mathrm{u})$ remains continuous, though its imaginary part may still differ from the imaginary part of $\ln f(u)$ by a multiple of $2 \pi$. This is corrected for in (48).

To monitor the discontinuity of the characteristic function Broadie and Kaya would have had to use a very fine discretisation of the Fourier integral leading to the cumulative density function. The method we propose opens up the possibility of using more advanced quadrature schemes, hereby speeding up their exact simulation algorithm considerably. Nonetheless, we expect that for highly path-dependent exotics biased simulation schemes such as the full truncation Euler scheme of Lord et al. [2006] and the quasi second-order scheme of Kahl and Jäckel [2005b] will remain the simulation schemes of choice. 


\subsection{Matytsin's SVJJ model}

In an unpublished but often referred to paper, Matytsin [1999] proposed his SVJJ model, which is an extension of the Bates [1996] model allowing for simultaneous jumps in both the stock price and the volatility. As Gatheral [2005] puts it, it is economically plausible that when the stock price jumps, the volatility has an upward jump, as "large moves follow large moves and small moves follow small moves". The model is able to generate extreme short-dated implied volatility skews, which is consistent with what we observe in the market.

In the notation we used for the Heston model, Matytsin's model can be specified as:

$$
\begin{aligned}
& \mathrm{dS}(\mathrm{t})=\left(\mu(\mathrm{t})-\lambda \bar{\mu}_{\mathrm{J}}\right) \mathrm{S}(\mathrm{t}) \mathrm{dt}+\sqrt{\mathrm{v}(\mathrm{t})} \mathrm{S}(\mathrm{t}) \mathrm{dW}_{\mathrm{S}}(\mathrm{t})+\mathrm{J}_{\mathrm{N}(\mathrm{t})} \mathrm{S}(\mathrm{t}) \mathrm{dN}(\mathrm{t}) \\
& \mathrm{dv}(\mathrm{t})=-\kappa(\mathrm{v}(\mathrm{t})-\theta) d \mathrm{t}+\omega \sqrt{\mathrm{v}(\mathrm{t})} \mathrm{dW}_{\mathrm{v}}(\mathrm{t})+\mathrm{v}_{\mathrm{J}} \mathrm{dN}(\mathrm{t})
\end{aligned}
$$

where $\mathrm{N}(\mathrm{t})$ is a Poisson process with intensity $\lambda$, independent of both Brownian motions. The random variable $J_{i}$ denotes the $i^{\text {th }}$ relative jump size and is lognormally distributed, i.e. $\ln \mathrm{J}_{\mathrm{i}} \sim \mathrm{N}\left(\mu_{\mathrm{J}}, \sigma_{\mathrm{J}}^{2}\right)$. Finally, $\mathrm{v}_{\mathrm{J}}$ is a constant. If the $\mathrm{i}^{\text {th }}$ jump occurs at time $\mathrm{t}$, the following happens:

- $\mathrm{S}(\mathrm{t}+)=\mathrm{J}_{\mathrm{i}} \mathrm{S}(\mathrm{t}-)$

- $\quad \mathrm{v}(\mathrm{t}+)=\mathrm{v}(\mathrm{t}-)+\mathrm{v}_{\mathrm{J}}$

The correction term in the drift of the stock price ensures no arbitrage. Hence, $\bar{\mu}_{\mathrm{J}}$ has to equal the expected relative jump size, and can be expressed in terms of $\mu_{\mathrm{J}}$ and $\sigma_{\mathrm{J}}{ }^{2}$ as:

$$
1+\bar{\mu}_{\mathrm{J}}=\mathbb{E}\left[\mathrm{J}_{\mathrm{i}}\right]=\exp \left(\mu_{\mathrm{J}}+\frac{1}{2} \sigma_{\mathrm{J}}^{2}\right)
$$

Denoting the Heston characteristic function as in section 5.2, it can be shown that the characteristic function in Matytsin's SVJJ model equals:

$$
\begin{aligned}
& \phi_{\mathrm{SvJJ}}\left(\mathrm{u}, \mathrm{S}(0), \mathrm{v}(0), \kappa, \omega, \theta, \rho, \lambda, \mu_{\mathrm{J}}, \sigma_{\mathrm{J}}, \tau\right)=\phi_{\mathrm{H}}(\mathrm{u}, \mathrm{S}(0), \mathrm{v}(0), \kappa, \omega, \theta, \rho, \tau) \\
& \cdot \exp \left(\lambda \tau\left(e^{i u \mu_{J}-\frac{1}{2} \sigma_{J}^{2} u^{2}} I(u, \tau)-1-i u \bar{\mu}_{J}\right)\right)
\end{aligned}
$$

with the unspecified term $\mathrm{I}(\mathrm{u}, \tau)$ equal to:

$$
\mathrm{I}(\mathrm{u}, \tau)=\frac{1}{\tau} \int_{0}^{\tau} \exp \left(\mathrm{v}_{\mathrm{J}} \mathrm{B}_{\mathrm{v}}(\mathrm{u}, \mathrm{s})\right) \mathrm{ds}=-\frac{2 \mathrm{v}_{\mathrm{J}}}{\tau \mathrm{p}_{+} \mathrm{p}_{-}} \int_{0}^{-\mathrm{v}_{\mathrm{J}} \mathrm{B}_{\mathrm{v}}(\mathrm{u}, \tau)} \frac{\mathrm{e}^{-\mathrm{z}}}{\left(1+\mathrm{z} / \mathrm{p}_{+}\right)\left(1+\mathrm{z} / \mathrm{p}_{-}\right)} \mathrm{dz}
$$

and $\mathrm{p}_{ \pm}=\mathrm{v}_{\mathrm{J}}(\beta(\mathrm{u}) \pm \mathrm{D}(\mathrm{u}))$. The functions $\mathrm{B}_{\mathrm{v}}, \beta$ and $\mathrm{D}$ are defined in section 2.1. Note the term $1 / \tau$ in the second integral which is erroneously omitted by both Matytsin and Gatheral [2005]. As $\mathrm{v}_{\mathrm{J}}$ or $\tau$ tends to zero, $\mathrm{I}(\mathrm{u}, \tau)$ tends to 1 and we recover Bates' model. Clearly in Bates' model the strip of regularity is the same as in Heston's model. The same holds for Matytsin's model, as for $\mathrm{u} \in \Lambda$ we know that $\mathrm{B}_{\mathrm{v}}(\mathrm{u}, \mathrm{s})$ for $0 \leq \mathrm{s} \leq \tau$ is finite, so that:

$$
|\mathrm{I}(\mathrm{u}, \tau)| \leq \max _{0 \leq \mathrm{s} \leq \tau} \exp \left(\mathrm{v}_{\mathrm{J}} \operatorname{Re}\left(\mathrm{B}_{\mathrm{v}}(\mathrm{u}, \mathrm{s})\right)\right)<\infty
$$


In this representation it is clear that we can immediately use formulation 2 to ensure that the characteristic function remains continuous. The downside of this representation is that for each value of $u$ a numerical integration is required to calculate $I(u, \tau)$, which would considerably slow down a calibration of this model. Unlike mentioned in Matytsin's paper or in Gatheral, the integral in (55) actually has a closed-form representation in terms of the exponential integral Ei:

$$
\begin{aligned}
\mathrm{I}(\mathrm{u}, \tau) & =\frac{2 \mathrm{v}_{\mathrm{J}}\left(\mathrm{f}\left(\mathrm{p}_{-}\right)-\mathrm{f}\left(\mathrm{p}_{+}\right)\right)}{\mathrm{p}_{+}-\mathrm{p}_{-}} \\
\mathrm{f}(\mathrm{p}) & =\mathrm{e}^{\mathrm{p}}\left\{\operatorname{Ei}(-\mathrm{p})-\operatorname{Ei}\left(-\mathrm{p}+\mathrm{v}_{\mathrm{J}} \mathrm{B}_{\mathrm{v}}(\mathrm{u}, \tau)\right)\right\}
\end{aligned}
$$

where the exponential integral is defined as:

$$
\begin{aligned}
\operatorname{Ei}(z) & =\gamma+\ln z-\operatorname{Ein}(-z) \\
\operatorname{Ein}(z) & =\int_{0}^{z} t^{-1}\left(1-e^{-t}\right) d t
\end{aligned}
$$

with $\gamma$ equal to the Euler-Mascheroni constant. Note that the modified exponential integral Ein(x) is an entire function.

It certainly seems worthwhile to explore whether evaluating $\mathrm{I}(\mathrm{u}, \tau)$ as in (57) is faster than the numerical integration proposed by Matytsin in equation (56). The crucial point, as in the rest of our paper, is to ensure that evaluating $\mathrm{I}(\mathrm{u}, \tau)$ with the closed-form expression returns the same value as we would have obtained from the numerical integration in (55). Press, Teukolsky, Vetterling and Flannery [1992] give an algorithm for the evaluation of the exponential integral, which switches between a power series and an asymptotic expansion depending on the size of its argument. The power series is based on a Taylor expansion of Ein(z):

$$
\operatorname{Ein}_{\text {power }}(z)=\sum_{j=1}^{\infty} \frac{(-1)^{j} z^{j}}{j \cdot j !}
$$

The asymptotic expansion is given by:

$$
\mathrm{Ei}_{\text {asymp }}(\mathrm{z}) \sim \frac{\mathrm{e}^{\mathrm{z}}}{\mathrm{z}} \cdot\left(1+\frac{1 !}{\mathrm{z}}+\frac{2 !}{\mathrm{z}^{2}}+\ldots\right)
$$

Though Press et al. [1992] only state their algorithm for $\mathrm{z}>0$, we can use the power series and the asymptotic expansion for complex-valued $\mathrm{z}$ if care is taken. For small values of $|\mathrm{z}|$, the power series is appropriate, whereas for large values of $|z|$ one should use the asymptotic expansion. It turns out that if we evaluate both exponential integral terms in either $f\left(p_{+}\right)$or $f\left(p_{-}\right)$with the same series, complex discontinuities can be avoided. The following theorem states this more precisely.

\section{Theorem 6}

Let $\mathrm{u} \in \Lambda_{\mathrm{x}}$ and assume the restrictions imposed in theorem 2 hold. When using the closed-form representation of $\mathrm{I}(\mathrm{u}, \tau)$ in Matytsin's SVJJ model we have two options when it comes to the evaluation of $\mathrm{f}\left(\mathrm{p}_{ \pm}\right)$in (57), if we want to avoid any complex discontinuities automatically. If we use the power series expansion, we should evaluate $\mathrm{f}\left(\mathrm{p}_{ \pm}\right)$as: 


$$
\begin{aligned}
& \mathrm{f}\left(\mathrm{p}_{+}\right)=\mathrm{e}^{\mathrm{p}_{+}}\left\{\ln \psi_{2}(\mathrm{u}, \tau)+\operatorname{Ein}_{\text {power }}\left(\mathrm{p}_{+}\right)-\operatorname{Ein}_{\text {power }}\left(\mathrm{p}_{+}-\mathrm{v}_{\mathrm{J}} \mathrm{B}_{\mathrm{v}}(\mathrm{u}, \tau)\right)\right\} \\
& \mathrm{f}\left(\mathrm{p}_{-}\right)=\mathrm{e}^{\mathrm{p}_{-}}\left\{\ln \psi_{2}(\mathrm{u}, \tau)+\mathrm{D}(\mathrm{u}) \tau+\operatorname{Ein}_{\text {power }}\left(-\mathrm{p}_{-}\right)-\operatorname{Ein}_{\text {power }}\left(-\mathrm{p}_{-}+\mathrm{v}_{\mathrm{J}} \mathrm{B}_{\mathrm{v}}(\mathrm{u}, \tau)\right)\right\}
\end{aligned}
$$

where we restrict the logarithm to its principal branch. When using the asymptotic expansion, $f(p)$ should be evaluated as:

$$
\mathrm{f}(\mathrm{p})=\mathrm{e}^{\mathrm{p}}\left\{\mathrm{Ei}_{\text {asymp }}(-\mathrm{p})-\mathrm{Ei}_{\text {asymp }}\left(-\mathrm{p}+\mathrm{v}_{\mathrm{J}} \mathrm{B}_{\mathrm{v}}(\mathrm{u}, \tau)\right)\right\}
$$

The only exception to these formulae is the situation where $\mathrm{D}= \pm \beta$, where we have $\mathrm{I}(\mathrm{u}, \tau)=1$.

\section{Proof:}

We first handle the exception, which follows immediately by noting that:

$$
\lim _{D \rightarrow \pm \beta} B_{v}(u, \tau)=0
$$

so that indeed $\mathrm{I}(\mathrm{u}, \tau)=1$. Continuing with the power series, first note that:

$$
\begin{aligned}
& -\mathrm{p}_{+}+\mathrm{v}_{\mathrm{J}} \mathrm{B}_{\mathrm{v}}(\mathrm{u}, \tau)=-\mathrm{p}_{+} \cdot \psi_{2}(\mathrm{u}, \tau)^{-1} \\
& -\mathrm{p}_{-}+\mathrm{v}_{\mathrm{J}} \mathrm{B}_{\mathrm{v}}(\mathrm{u}, \tau)=-\mathrm{p}_{-} \cdot \mathrm{e}^{-\mathrm{D}(\mathrm{u}) \tau} \psi_{2}(\mathrm{u}, \tau)^{-1}
\end{aligned}
$$

where we recognise a dependence on $\psi_{2}(\mathrm{u}, \tau)$, the term earlier defined in formulation 2 in (11). As we know how to keep its argument continuous for $\mathrm{u} \in \Lambda_{\mathrm{x}}$ and under the restrictions on the parameters and u imposed in theorem 2, the representation in (61) immediately follows. Since raising a complex number to a positive integer is not a multivalued operation, it is clear that the power series and the asymptotic expansion do not give rise to any complex discontinuities.

The only remaining thing we need to check is that the principal branch of the logarithm is the appropriate one here. We can write $\ln \psi_{2}(\mathrm{u}, \tau)$ as $\ln \psi_{2}(\mathrm{u}, \tau)+2 \pi \mathrm{mi}$ and $\ln \psi_{2}(\mathrm{u}, \tau)+2 \pi \mathrm{ni}$ in $\mathrm{f}\left(\mathrm{p}_{+}\right)$ and $\mathrm{f}\left(\mathrm{p}_{-}\right)$respectively, for integer $\mathrm{m}$ and $\mathrm{n}$, to check which is the appropriate branch. For $\mathrm{u} \in \Lambda_{\mathrm{x}}$ and $\mathrm{u}=\mathrm{yi}$ we know that $\mathrm{I}(\mathrm{u}, \tau)$ should be a real number. Furthermore, $\beta(\mathrm{yi})$ is real here, and $\mathrm{D}(\mathrm{yi})$ can be either purely real or purely imaginary. When $\mathrm{D}(\mathrm{yi})$ is real, we find:

$$
\operatorname{Im}(\mathrm{I}(\mathrm{yi}, \tau))=-\frac{2 \pi \mathrm{v}_{\mathrm{J}} \mathrm{e}^{\beta-\mathrm{D}_{\mathrm{r}}}\left(\mathrm{e}^{2 \mathrm{D}_{\mathrm{r}}} \mathrm{m}-\mathrm{n}\right)}{\mathrm{D}_{\mathrm{r}}}
$$

whereas when $\mathrm{D}(\mathrm{yi})$ is purely imaginary:

$$
\operatorname{Im}(\mathrm{I}(\mathrm{yi}, \tau))=-\frac{2 \pi \mathrm{v}_{\mathrm{J}} \mathrm{e}^{\beta} \sin \left(\mathrm{D}_{\mathrm{i}}\right)(\mathrm{m}+\mathrm{n})}{\mathrm{D}_{\mathrm{i}}}
$$

It follows immediately that both terms are guaranteed to be zero when $\mathrm{m}=\mathrm{n}=0$.

It is somewhat restrictive that we can only simultaneously use the power series or the asymptotic

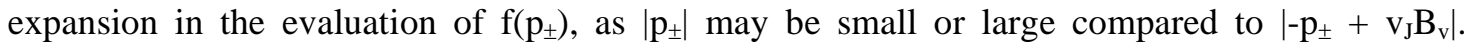
Consider the calculation of $\mathrm{f}(\mathrm{p})$, and suppose that $|\mathrm{p}|$ is such that we can use the asymptotic series, 
whereas $\left|-p+v_{J} B_{v}\right|$ is small enough for the power series to be used. In this case, we need to take the complex logarithm of $-\mathrm{p}+\mathrm{v}_{\mathrm{J}} \mathrm{B}_{\mathrm{v}}$, which will cross the negative real line for some relevant parameter values. This could be avoided if we had an asymptotic expansion for Ein(z). At present we are not aware of the existence of such an expansion, so that we leave this for future research. An alternative that could be considered is to relate the exponential integral Ei(z) to other related special functions, such as the exponential integral $E_{1}(z)$ and the incomplete gamma function $\Gamma(0, \mathrm{z})$. Unfortunately, it seems that algorithms for both special functions require the evaluation of a complex logarithm for some ranges of $\mathrm{z}$.

At present our conclusion is therefore that an implementation of Matytsin's model should first check whether both $f\left(p_{+}\right)$and $f\left(p_{-}\right)$can be evaluated using equations (61) and (62). If this is the case, the closed-form solution should be used, otherwise we recommend that $\mathrm{I}(\mathrm{u}, \tau)$ be calculated by numerical integration of the second integral in equation (55).

\section{Conclusions}

In this paper we have analysed the complex discontinuities which are found when evaluating the closed-form characteristic function of several popular option pricing models. Such discontinuities have first been documented in option pricing by Schöbel and Zhu [1999], and are, at least in the Heston and Schöbel-Zhu stochastic volatility models and their extensions, caused by the branch switching of the complex logarithm. Being unaware of these issues can lead to completely wrong option prices if we price European options by means of Fourier inversion.

When pricing options via Fourier inversion, the method which seems to be used by most practitioners to correct for these discontinuities is to carefully monitor the imaginary part of the complex logarithm and change its branch if a discontinuity is detected. Clearly this method is not foolproof, and moreover, highly inefficient. The only method to this date to guarantee a continuous characteristic function is to bypass the closed-form solution and numerically integrate the ordinary differential equation that gives rise to the complex logarithm. Unfortunately this approach forsakes the comparative advantage of these option pricing models, precisely the fact that their characteristic function can be calculated in closed-form.

As a foolproof alternative, Kahl and Jäckel [2005] recently proposed their rotation count algorithm, which is an easily implementable algorithm that claims to be able to keep the complex logarithm in the Heston model continuous. In this article we have rigorously proven, under nonrestrictive conditions on the parameters, that this is indeed the case. Our analysis has shown that the problem is intimately connected with the moment stability of the Heston model. Under the same conditions we have also been able to prove that in an alternative formulation of the Heston characteristic function, which has appeared in e.g. Duffie, Pan and Singleton [2000], Schoutens, Simons and Tistaert [2004] and Gatheral [2005], the principal branch of the complex logarithm is the correct one. As this formulation is easier to implement than the rotation count algorithm, and more numerically stable, it should be the preferred formulation.

With the lessons from the Heston model in hand, the remainder of this article investigates the complex discontinuities that arise in other models. First of all we show that although the Variance Gamma model involves the complex power function, its characteristic function can be evaluated by restricting the complex power function to its principal branch. Secondly, we have shown how to avoid complex discontinuities in both the Schöbel-Zhu model and the exact simulation algorithm of the Heston model proposed by Broadie and Kaya [2006]. Finally, we also considered Matytsin's [1999] extension of Heston's stochastic volatility model, the SVJJ model, which allows for simultaneous jumps in the underlying asset and the stochastic volatility process. We have shown that there is a closed-form solution for its characteristic function involving the exponential integral function. Unfortunately the complex discontinuities that arise from the branch switching of the exponential integral can only be avoided under particular circumstances. 


\section{Bibliography}

ANDERSEN, L.B.G. AND J. ANDREASEN (2002). “Volatile volatilities”, Risk, vol. 15, no. 12, December 2002, pp. 163-168.

ANDERSEN, L.B.G. AND R. BROTHERTON-RATCLIFFE (2005). "Extended LIBOR market models with stochastic volatility”, Journal of Computational Finance, vol. 9, no. 1, pp. 1-40.

Andersen, L.B.G. AND V.V. Piterbarg (2004). "Moment explosions in stochastic volatility models”, working paper, Bank of America.

BAKSHI, G., CAO, C. AND Z. CHEN (1997). "Empirical performance of alternative option pricing models”, Journal of Finance, vol. 52, pp. 2003-2049.

BATES, D.S. (1996). "Jumps and stochastic volatility: exchange rate processes implicit in Deutsche Mark options”, Review of Financial Studies, vol.9, no.1., pp. 69-107.

BROADIE, M. AND Ö. KAYA (2006). "Exact simulation of stochastic volatility and other affine jump diffusion processes”, Operations Research, vol. 54, no. 2, pp. 217-231.

CHENG, P. AND O. SCAILLET (2005). "Linear-quadratic jump-diffusion modelling with application to stochastic volatility”, working paper, Crédit Suisse, FAME and HEC Genève.

DUfFIE, D., PAN, J. AND K. SingLETON (2000). “Transform analysis and asset pricing for affine jumpdiffusions”, Econometrica, vol. 68, pp. 1343-1376.

GASPAR, R. (2004). "General quadratic term structures of bond, futures and forward prices”, SSE/EFI Working paper Series in Economics and Finance, no. 559.

Gatheral, J. (2005). Case studies in financial modelling course notes, Courant Institute of Mathematical Sciences, Fall Term 2005.

HESTON, S.L. (1993). "A closed-form solution for options with stochastic volatility with applications to bond and currency options”, Review of Financial Studies, vol. 6, no. 2, pp. 327-343.

JÄCKEL, P. (2004). "Stochastic volatility models: past, present and future”, pp. 379-390 in P. Wilmott (ed). The Best of Wilmott 1: Incorporating the Quantitative Finance Review, P. Wilmott (ed.), John Wiley and Sons.

KAHL, C. AND P. JÄCKEL (2005A). "Not-so-complex logarithms in the Heston model”, Wilmott Magazine, September 2005.

KAHL, C. AND P. JÄCKEL (2005B). "Fast strong approximation Monte-Carlo schemes for stochastic volatility models", working paper, ABN·AMRO and University of Wuppertal.

LEE, R.W. (2004). "Option pricing by transform methods: extensions, unification and error control”, Journal of Computational Finance, vol. 7, no. 3, pp. 51-86.

LEWIS, A. (2001). "A simple option formula for general jump-diffusion and other exponential Lévy processes”, working paper, OptionCity.net. 
LORD, R. AND C. KAHL (2006). “Optimal Fourier inversion in semi-analytical option pricing”, working paper, Erasmus University Rotterdam, Rabobank International, University of Wuppertal and ABN·AMRO.

LORD, R., KOEKKOEK, R. AND D. VAN DIJK (2006). "A comparison of biased simulation schemes for stochastic volatility models”, working paper, Erasmus University Rotterdam, Rabobank International and Robeco Alternative Investments.

MADAN, D.B. AND E. SENETA (1990). “The variance gamma (V.G.) model for share market returns”, Journal of Business, vol. 63, no. 4, pp. 511-524.

MAtytsin, A. (1999). “Modelling volatility and volatility derivatives", Columbia Practitioners Conference on the Mathematics of Finance.

Mercurio, F. AND N. MoReni (2006). "Inflation with a smile”, Risk, vol. 19, no. 3, March 2006, pp. 70-75.

Press, W.H., Teukolsky, S.A., VetTerling, W.T. And B.P. Flannery (1992). Numerical Recipes in $C, 2^{\text {nd }}$ edition, Cambridge University Press.

SCHÖBEL, R. AND J. ZHU (1999). "Stochastic volatility with an Ornstein-Uhlenbeck process: an extension”, European Finance Review, vol. 3, pp. 23-46.

SCHOUTENS, W., E. Simons AND J. Tistaert (2004). “A perfect calibration! Now what?”, Wilmott Magazine, March 2004.

STEIN, E. AND J. STEIN (1991). "Stock-price distributions with stochastic volatility - an analytic approach”, Review of Financial Studies, vol. 4, pp. 727-752.

ZHU, J. (2000). Modular pricing of options - an application of Fourier analysis, Lecture notes in economics and mathematical systems, no. 493, Springer Verlag. 


\section{Appendix - Proofs}

In this appendix we provide the proofs for lemma's 2 and 3.

\section{Lemma 2}

Consider a continuous function $\mathrm{z}: \mathbb{R} \rightarrow \mathbb{C}$, where both the real and complex part of $\mathrm{z}$ are strictly monotone. Assume that $\mathrm{z}$ never passes through the origin. Adding $\mathrm{y} \in \mathbb{R}$ where $\mathrm{y} \neq 0$ to $\mathrm{z}(\mathrm{x})$ does not add any discontinuities to the principal argument of $\mathrm{z}(\mathrm{x})+\mathrm{y}$ when compared to the principal argument of $\mathrm{z}(\mathrm{x})$, if and only if:

- $\operatorname{Re}(\mathrm{z}(\mathrm{x})) \notin(-\mathrm{y}, 0)$ for $\mathrm{y}>0$ whenever $\operatorname{Im}(\mathrm{z}(\mathrm{x}))$ changes sign;

- $\operatorname{Re}(\mathrm{z}(\mathrm{x})) \notin(0,-\mathrm{y})$ for $\mathrm{y}<0$ whenever $\operatorname{Im}(\mathrm{z}(\mathrm{x}))$ changes sign.

\section{Proof:}

Let us first define the difference of the principal arguments of $\mathrm{z}(\mathrm{x})$ and $\mathrm{z}(\mathrm{x})+\mathrm{y}$ as $\mathrm{f}(\mathrm{x})$ :

$$
\mathrm{f}(\mathrm{x})=\arg (\mathrm{z}(\mathrm{x}))-\arg (\mathrm{z}(\mathrm{x})+\mathrm{y})
$$

Let a trajectory from quadrant $i$ to quadrant $j$, without crossing any quadrants inbetween, be denoted as a tuple $(\mathrm{i}, \mathrm{j})$. The direction in which the trajectory is traversed does not matter here. Trajectories of $\mathrm{z}$ that do not cause any discontinuities are $(1,2),(1,4),(3,4)$. A trajectory of $\mathrm{z}$ that does cause discontinuities is $(2,3)$. Clearly the horizontal trajectories $(1,2),(3,4)$ and vice versa can be neglected here, as $\operatorname{Im}(\mathrm{z}(\mathrm{x}))$ does not change sign here. The diagonal trajectories $(1,3)$ and $(2,4)$ can also be excluded, as we assumed that $\mathrm{z}$ never passes through the origin. Finally, let $\mathrm{x}^{*}$ be that $\mathrm{x}$ on the trajectory such that $\operatorname{Im}\left(\mathrm{z}\left(\mathrm{x}^{*}\right)\right)=0$.

Let us start with the trajectory $(1,4)$. Evidently the trajectory of $\mathrm{z}(\mathrm{x})+\mathrm{y}$ remains $(1,4)$, provided that $\operatorname{Re}\left(\mathrm{z}\left(\mathrm{x}^{*}\right)\right)+\mathrm{y}>0$. However, if $\operatorname{Re}\left(\mathrm{z}\left(\mathrm{x}^{*}\right)\right)+\mathrm{y}<0$, the trajectory will pass through the origin in an infinitesimal neighbourhood of $x^{*}$. This will lead to a discontinuity in the principal argument, so we have to exclude this case. The same happens when $\operatorname{Re}\left(\mathrm{z}\left(\mathrm{x}^{*}\right)\right)+\mathrm{y}=0$.

If we start out with $(2,3)$ as the trajectory of $z(x)$, the same analysis leads to the requirement that $\operatorname{Re}\left(\mathrm{z}\left(\mathrm{x}^{*}\right)\right)+\mathrm{y}<0$, if we want to keep $\mathrm{f}(\mathrm{x})$ continuous. Collecting the results we find that $\mathrm{f}(\mathrm{x})$ remains continuous provided that:

- If $\operatorname{Re}\left(\mathrm{z}\left(\mathrm{x}^{*}\right)\right)<0$, y must satisfy $\operatorname{Re}\left(\mathrm{z}\left(\mathrm{x}^{*}\right)\right)+\mathrm{y}<0$;

- $\quad$ If $\operatorname{Re}\left(\mathrm{z}\left(\mathrm{x}^{*}\right)\right)>0$, y must satisfy $\operatorname{Re}\left(\mathrm{z}\left(\mathrm{x}^{*}\right)\right)+\mathrm{y}>0$.

This result is slightly rephrased in the theorem, so this concludes the proof.

\section{Lemma A.1}

If $\mathrm{x}, \mathrm{y} \in \mathbb{C}$ and $\mathrm{y}=\sqrt{\mathrm{x}}$, with $\mathrm{y}_{\mathrm{r}}>0$, we can write:

$$
y_{r}=\sqrt{\frac{1}{2} x_{r}+\frac{1}{2} \sqrt{x_{r}^{2}+x_{i}^{2}}} \quad y_{i}=\frac{x_{i}}{2 y_{r}}
$$

if we use the convention that the real part of the square root is positive. 


\section{Lemma A.2}

For $\mathrm{x}, \mathrm{y} \in \mathbb{C}$ we have that if $\operatorname{Re}(\mathrm{x}) \operatorname{Re}(\sqrt{\mathrm{y}})-\operatorname{Im}(\mathrm{x}) \operatorname{Im}(\sqrt{\mathrm{y}}) \geq 0$, then $\mathrm{x} \sqrt{\mathrm{y}}=\sqrt{\mathrm{x}^{2} \mathrm{y}}$. If the condition is not satisfied, we have $x \sqrt{y}=-\sqrt{x^{2} y}$.

\section{Lemma A.3}

For $\mathrm{x}, \mathrm{y} \in \mathbb{C}$ consider the following complex number:

$$
z=\frac{x-\sqrt{x^{2}+y}}{x+\sqrt{x^{2}+y}}
$$

Its modulus is equal to 1 if $\mathrm{x}=0$ or $\operatorname{Re}\left(\mathrm{y} / \mathrm{x}^{2}\right) \leq-1$ and $\operatorname{Im}\left(\mathrm{y} / \mathrm{x}^{2}\right)=0$, and less than 1 if:

$$
\operatorname{Re}\left(\frac{1}{x}\right) \operatorname{Re}\left(\sqrt{x^{2}+y}\right)-\operatorname{Im}\left(\frac{1}{x}\right) \operatorname{Im}\left(\sqrt{x^{2}+y}\right) \geq 0
$$

\section{Proof:}

If $\mathrm{x}=0$ we immediately have $\mathrm{z}=-1$. Assume that (A.4) holds true and that $\mathrm{x} \neq 0$. Then:

$$
z=\frac{x-\sqrt{x^{2}+y}}{x+\sqrt{x^{2}+y}}=\frac{1-\frac{1}{x} \sqrt{x^{2}+y}}{1+\frac{1}{x} \sqrt{x^{2}+y}}=\frac{1-\sqrt{1+\frac{y}{x^{2}}}}{1+\sqrt{1+\frac{y}{x^{2}}}}
$$

by virtue of lemma A.2. We can write this as $\mathrm{z}=(1-\mathrm{u}) /(1+\mathrm{u})$ with $\mathrm{u}_{\mathrm{r}} \geq 0$. Its modulus satisfies:

$$
|\mathrm{z}|^{2}=\left|\frac{1-\mathrm{u}}{1+\mathrm{u}}\right|^{2}=\frac{\left(1-\mathrm{u}_{\mathrm{r}}\right)^{2}+\mathrm{u}_{\mathrm{i}}^{2}}{\left(1+\mathrm{u}_{\mathrm{r}}\right)^{2}+\mathrm{u}_{\mathrm{i}}^{2}} \leq 1
$$

with equality attained only when $\mathrm{u}_{\mathrm{r}}=0$, i.e. if and only if $\operatorname{Re}\left(\mathrm{y} / \mathrm{x}^{2}\right) \leq-1$ and $\operatorname{Im}\left(\mathrm{y} / \mathrm{x}^{2}\right)=0$. Clearly the modulus of $\mathrm{z}$ is larger than or equal to 1 if (A.4) does not hold true.

The following proposition collects many properties of some functions that we require hereafter. All properties can be proven by using basic algebra, so that we omit the proof.

\section{Proposition}

Consider the following two functions:

$$
\begin{aligned}
& p(x, y)=\operatorname{Re}\left(D(u)^{2}\right)=\kappa^{2}+\omega^{2}\left(1-\rho^{2}\right) x^{2}-\omega(\omega-2 \kappa \rho) y-\omega^{2}\left(1-\rho^{2}\right) y^{2} \\
& q(x, y)=\operatorname{Im}\left(D(u)^{2}\right)=\omega(\omega-2 \kappa \rho) x+2 \omega^{2}\left(1-\rho^{2}\right) x y
\end{aligned}
$$

where we have introduced the convention that $\mathrm{u}=\mathrm{x}+\mathrm{yi}$, for $\mathrm{x}, \mathrm{y} \in \mathbb{R}$. Furthermore, define:

$$
y_{1}=-\frac{\omega-2 \kappa \rho}{2 \omega\left(1-\rho^{2}\right)} \quad y_{2}=-\frac{\kappa}{\rho \omega}
$$

Note that: 
- when $\rho<0$ or $\rho \geq 2 \kappa / \omega, \mathrm{y}_{2} \geq \mathrm{y}_{1}$;

- when $0<\rho \leq 2 \kappa / \omega, \mathrm{y}_{2} \leq \mathrm{y}_{1}$.

For $\mathrm{x} \geq 0$ the function $\mathrm{p}$ has the following properties:

- $\quad \mathrm{p}$ is maximal w.r.t. $\mathrm{y}$ in $\mathrm{y}_{1}$;

- For $\mathrm{y}<\mathrm{y}_{1}, \mathrm{p}$ is strictly increasing in $\mathrm{y}$, for $\mathrm{y}>\mathrm{y}_{1}$, p is strictly decreasing in $\mathrm{y}$;

- $\quad \mathrm{p}$ is always strictly increasing in $\mathrm{x}$;

Similarly, we can show that for $\mathrm{x} \geq 0$ the function $\mathrm{q}$ has the following properties:

- $\mathrm{q}\left(\mathrm{x}, \mathrm{y}_{1}\right)=0$;

- $\mathrm{q}$ is positive and strictly increasing in $\mathrm{x}$ for $\mathrm{y}>\mathrm{y}_{1}$;

- $\quad \mathrm{q}$ is negative and strictly decreasing in $\mathrm{x}$ for $\mathrm{y}<\mathrm{y}_{1}$;

- $\mathrm{q}$ is strictly increasing in $\mathrm{y}$.

The following lemma is key to proving lemma 3. Its proof uses many of the previous properties.

\section{Lemma A.4}

For $\mathrm{x} \geq 0$, the functions $\mathrm{p}$ and $\mathrm{q}$ defined in the previous proposition satisfy:

$$
(\kappa+\omega \rho y)\left(p(x, y)+\sqrt{p(x, y)^{2}+q(x, y)^{2}}\right)-\omega \rho x q(x, y) \geq 0
$$

if in addition $\rho \leq \kappa / \omega$, or $\mathrm{y} \geq \mathrm{y}_{2}$ and $\kappa / \omega \leq \rho \leq 2 \kappa / \omega$.

\section{Proof:}

We split the proof into four parts: $\rho<0, \rho=0,0<\rho \leq \kappa / \omega$ and $\kappa / \omega \leq \rho \leq 2 \kappa / \omega$. Furthermore, within each case we will divide the problem into several sub cases, based on ranges for the variable y.

\section{Case 1: $\rho<0$}

1a) $\mathbf{y} \leq \mathrm{y}_{1} \leq \mathrm{y}_{2}$

We can reshuffle:

$$
(\kappa+\omega \rho y) \sqrt{p(x, y)^{2}+q(x, y)^{2}} \geq-(\kappa+\omega \rho y) p(x, y)+\omega \rho x q(x, y)
$$

Since $\mathrm{y}_{1} \leq \mathrm{y}_{2}$ here, we have $\kappa+\omega \rho y \geq 0$, so that it is sufficient to square both sides and prove the resulting inequality. We obtain the following inequality:

$$
\begin{aligned}
& \omega^{2} x q(x, y) f(x, y) \geq 0 \\
& f(x, y)=\omega \rho(2 \kappa-\omega \rho)\left(x^{2}+y^{2}\right)+\kappa^{2}(1+2 y)
\end{aligned}
$$

Since $\mathrm{q}$ is negative for $\mathrm{y} \leq \mathrm{y}_{1}$, we have to prove that $\mathrm{f}(\mathrm{x}, \mathrm{y}) \leq 0$. The function $\mathrm{f}$ is maximal with respect to $\mathrm{x}$ for $\mathrm{x}=0$, and maximal w.r.t. $\mathrm{y}$ in:

$$
\mathrm{y}_{3} \equiv \frac{\kappa^{2}}{-\omega \rho(2 \kappa-\omega \rho)}
$$


Here we have $y_{3} \geq 0$. Since $y_{1} \leq 0$, it suffices to show that $f\left(0, y_{1}\right)<0$. We have:

$$
\frac{4 \omega\left(1-\rho^{2}\right)^{2}}{\rho} f\left(0, y_{1}\right)=(2 \kappa-\omega \rho)\left((2 \kappa-\omega \rho)^{2}+\omega^{2}\left(1-\rho^{2}\right)\right) \equiv g(\rho)
$$

Clearly, $\mathrm{g}(\rho)>0$ for $\rho<\min (2 \kappa / \omega, 1)$. Since the left-hand side is negative for $\rho<0, \mathrm{f}\left(0, \mathrm{y}_{1}\right)<0$.

1b) $\mathrm{y}_{1} \leq \mathrm{y} \leq \mathrm{y}_{2}$

In this region we still have $\kappa+\omega \rho y \geq 0$. If $p$ is positive, the inequality is immediately seen to be true. If $\mathrm{p}$ is negative, we have $\sqrt{\mathrm{p}(\mathrm{x}, \mathrm{y})^{2}+\mathrm{q}(\mathrm{x}, \mathrm{y})^{2}} \geq-\mathrm{p}(\mathrm{x}, \mathrm{y})$, so that the inequality clearly also holds.

1c) $y_{1} \leq y_{2} \leq y$

Here $\kappa+\omega \rho y \leq 0$. First note that in this region:

$$
(\kappa+\omega \rho y) p(x, y)-\omega \rho x q(x, y) \geq 0
$$

If $p$ is negative, the proof is easy, since $\rho \leq 0$ and $q$ is nonnegative. So let us assume that $p$ is positive. Working out the function shows that $\mathrm{x}^{2}$ is the only power of $\mathrm{x}$ in it, and its coefficient is:

$$
\omega^{2}\left(\kappa\left(1+\rho^{2}\right)-\omega \rho\left(1+\left(1-\rho^{2}\right) y\right)\right)
$$

which is increasing in $y$, and positive for $y \geq 0$. Since $y_{2} \geq 0$ in this region, the coefficient of $x^{2}$ is positive. Because $\mathrm{p}$ too is strictly increasing in $\mathrm{x}$, it suffices to check the inequality for that $\mathrm{x}$ where $\mathrm{p}(\mathrm{x}, \mathrm{y})=0$. But then the remaining inequality is $-\omega \rho \mathrm{xq}(\mathrm{x}, \mathrm{y}) \geq 0$ which is immediately seen to be true. Hence, if we can prove the inequality from 1a) but now in reverse, we are done:

$$
\omega^{2} x q(x, y) f(x, y) \leq 0
$$

Since $q$ is here positive, it remains to show that $f(x, y)$ is negative. As in $1 a), f$ is maximal w.r.t. $x$ in $\mathrm{x}=0$ and maximal w.r.t. $\mathrm{y}$ in $\mathrm{y}_{3}$. In this region $\mathrm{y}_{2} \geq \mathrm{y}_{3}$, so that it is sufficient to check that $f\left(0, y_{2}\right) \leq 0$. It turns out that $f\left(0, y_{2}\right)=0$, which concludes the proof of case 1 .

\section{Case 2: $\rho=0$}

When $\rho=0$, the inequality can be reduced to $p(x, y)+\sqrt{p(x, y)^{2}+q(x, y)^{2}} \geq 0$. Using the rationale of $1 \mathrm{~b}$ ) it is clear that this is true.

\section{Case 3: $0 \leq \rho \leq \kappa / \omega$}

\section{3a) $\mathbf{y} \leq \mathbf{y}_{2} \leq \mathrm{y}_{1}$}

In this region we have $\kappa+\omega \rho y \leq 0$. We should be able to follow the proof of $1 \mathrm{c}$ ) here, in particular we can show that (A.14) is true. Namely, if p is negative, we are immediately ready as q is negative. So let us assume that $p$ is positive. The coefficient of $x^{2}$ is as in (A.15), which in this region of the proof means it is decreasing in $\mathrm{y}$. For $\mathrm{y}=\mathrm{y}_{2}$ it is still positive. We can therefore use the same proof of (A.14) as in 1c). 
Subsequently, we have to prove (A.16) again. As q is negative here, we have to prove that $\mathrm{f}$ is nonnegative. The function $\mathrm{f}$ is here minimal w.r.t. $\mathrm{x}$ in $\mathrm{x}=0$, and minimal w.r.t. $\mathrm{y}$ in $\mathrm{y}_{3}$. Since $\mathrm{y}_{2}$ $\leq \mathrm{y}_{3}$ in this region, it suffices to check that $\mathrm{f}\left(0, \mathrm{y}_{2}\right) \geq 0$. But $\mathrm{f}\left(0, \mathrm{y}_{2}\right)=0$, so we are done.

\section{3b) $\mathbf{y}_{2} \leq \mathbf{y} \leq \mathbf{y}_{1}$}

As $\kappa+\omega \rho y \geq 0$, and $\rho$ and q both have a different sign here as compared to $1 \mathrm{~b}$ ), we can use the exact same line of reasoning to prove the inequality in this region.

\section{3c) $\mathbf{y}_{2} \leq \mathrm{y}_{1} \leq \mathrm{y}$}

Again, in this region we have $\kappa+\omega \rho y \geq 0$, and we can use the same line of proof as in $1 \mathrm{a})$. We have to prove (A.11). As $q$ is nonnegative here, it remains to prove that $\mathrm{f}$ is nonnegative too. Again $\mathrm{f}$ is minimal w.r.t. $\mathrm{x}$ in $\mathrm{x}=0$ and minimal w.r.t. $\mathrm{y}$ in $\mathrm{y}_{3}$. We can prove that $\mathrm{y}_{1}>\mathrm{y}_{3}$, namely:

$$
y_{1}-y_{3}=\frac{\kappa^{2}+(\kappa-\omega \rho)^{2}+2 \kappa \rho^{2}(\kappa-\omega \rho)}{2 \omega \rho(2 \kappa-\omega \rho)\left(1-\rho^{2}\right)}>0
$$

It is therefore sufficient to check that $f\left(0, y_{1}\right)>0$. From (A.13) we already know that $g(\rho)>0$ in this region, so that it is immediately clear that $\mathrm{f}\left(0, \mathrm{y}_{1}\right)>0$.

\section{Case 4: $\kappa / \omega \leq \rho \leq 2 \kappa / \omega$}

In this region for $\rho$ it seems that only for $y \geq y_{2}$ we can prove the original inequality (A.9). Note that here we always have $\kappa+\omega \rho y \geq 0$.

4a) $\mathbf{y}_{2} \leq \mathbf{y} \leq \mathbf{y}_{1}$

As $\kappa+\omega \rho y \geq 0$, and $\rho$ and q both have a different sign here as compared to $1 \mathrm{~b}$ ), we can use the exact same line of reasoning to prove the inequality in this region.

\section{4b) $\mathbf{y} \geq \mathbf{y}_{1}$}

We again use the same analysis as in $3 c$ ). We can assume that $\kappa<\omega$ because otherwise we would already have the proof for $\rho \leq 1$ from the previous analysis. Note that the proof from 3c) is still valid so that $y_{1} \geq y_{3}$. Hence, it is sufficient to check that $f\left(0, y_{1}\right) \geq 0$. From case $\left.1 a\right)$ we know that $\mathrm{g}(\rho)>0$ for $\rho<\max (2 \kappa / \omega, 1)$ and in fact $\mathrm{g}(2 \kappa / \omega)=0$ and $\mathrm{g}(1)=(2 \kappa-\omega)^{3}>0$.

We now have the necessary machinery to prove lemma 3.

\section{Lemma 3}

If $\rho \leq \kappa / \omega$, or $\operatorname{Im}(u) \geq y_{2}$ and $\kappa / \omega \leq \rho \leq 2 \kappa / \omega$, we have:

$$
|G(u)|=\left|\frac{\beta(u)-D(u)}{\beta(u)+D(u)}\right| \leq 1
$$

where the inequality is strict, except for some cases where $\operatorname{Re}(u)=0$ or $\rho=2 \kappa / \omega$.

\section{Proof:}

As before, we will write $u=x+$ yi here. It is fairly easy to show that $G(-\bar{u})=\overline{G(u)}$, so that it suffices to focus on the case $x \geq 0$. Since $D^{2}=\beta^{2}+\omega^{2} u(i+u), G(u)$ is of the form treated in lemma 
A.3. Let us therefore first assume that $\beta \neq 0$. The condition from lemma A.3 which guarantees that (A.18) holds, is then:

$$
\operatorname{Re}(\beta) \operatorname{Re}(D)+\operatorname{Im}(\beta) \operatorname{Im}(D) \geq 0
$$

which we obtain from (A.4) by multiplying with $|\beta|^{2}$. In the proof we will often require the functions $\mathrm{p}$ and $\mathrm{q}$ we introduced earlier in our proposition, so that we repeat their definitions here:

$$
\begin{aligned}
& \mathrm{p}(\mathrm{x}, \mathrm{y})=\operatorname{Re}\left(\mathrm{D}(\mathrm{u})^{2}\right)=\kappa^{2}+\omega^{2}\left(1-\rho^{2}\right) \mathrm{x}^{2}-\omega(\omega-2 \kappa \rho) y-\omega^{2}\left(1-\rho^{2}\right) \mathrm{y}^{2} \\
& \mathrm{q}(\mathrm{x}, \mathrm{y})=\operatorname{Im}\left(\mathrm{D}(\mathrm{u})^{2}\right)=\omega(\omega-2 \kappa \rho) \mathrm{x}+2 \omega^{2}\left(1-\rho^{2}\right) \mathrm{xy}
\end{aligned}
$$

We now split the proof into several cases.

\section{Case 1: $x \geq 0, \beta \neq 0, \operatorname{Re}(D)=\mathbf{0}$}

Lemma A.1 shows us that:

$$
\operatorname{Re}(\mathrm{D}(\mathrm{u}))=\sqrt{\frac{1}{2} \mathrm{p}(\mathrm{x}, \mathrm{y})^{2}+\frac{1}{2} \sqrt{\mathrm{p}(\mathrm{x}, \mathrm{y})^{2}+\mathrm{q}(\mathrm{x}, \mathrm{y})^{2}}}
$$

If $\operatorname{Re}(D)=0$ we must therefore have $q=\operatorname{Im}\left(D^{2}\right)=0$ and $p=\operatorname{Re}\left(D^{2}\right) \leq 0$. Since $D=\sqrt{p+q i}$, it is clear that we then have $\mathrm{D}=\mathrm{i} \sqrt{-\mathrm{p}}$, and (A.19) becomes:

$$
-\omega \rho x \sqrt{-p(x, y)} \geq 0
$$

Clearly q can only be zero if $\mathrm{x}=0$ or if $\mathrm{y}=\mathrm{y}_{1}$. When $\mathrm{x}=0$, (A.22) clearly holds true. In the second case, note that:

$$
p\left(x, y_{1}\right)=\frac{(2 \kappa-\omega \rho)^{2}+\omega^{2}\left(1-\rho^{2}\right)+4 \omega^{2}\left(1-\rho^{2}\right)^{2} x^{2}}{4\left(1-\rho^{2}\right)}
$$

For $\mathrm{x}>0$ and $|\rho|<1$ this is always strictly positive, so that (A.19) always holds in case 1.

\section{Case 2: $x \geq 0, \beta \neq 0, \operatorname{Re}(D)>0$}

Since $\operatorname{Re}(\mathrm{D})>0$, we can multiply (A.19) by $2 \operatorname{Re}(\mathrm{D})$ and apply lemma A.1 to obtain:

$$
(\kappa+\omega \rho y)\left(p(x, y)+\sqrt{p(x, y)^{2}+q(x, y)^{2}}\right)-\omega \rho x q(x, y) \geq 0
$$

This inequality is proven to be true in lemma A.4 for the following cases:

- $\rho \leq \kappa / \omega$ and $y \in \mathbb{R}$;

- $\kappa / \omega \leq \rho \leq 2 \kappa / \omega$ and $y \geq y_{2}$.

\section{Case 3: $x \geq 0, \beta=0$}

When $\beta=0$, lemma A.3 states that $|G|=1$. We can only have $\beta=0$ when $\rho \neq 0, x=0$ and $y=y_{2}$. 
All that remains to be checked is that the inequality in (A.18) can only become an equality when $\mathrm{x}=0$. In case 3 this has already been concluded. The only possibility in case 1 was indeed $x=0$, and we can have $|G|=1$ in case 1 , provided that $\operatorname{Im}(\beta)=0$ or $\operatorname{Im}(D)=0$. Hence, we have to check that in the cases analysed under case 2, the condition stated in lemma A.3 cannot occur for $\mathrm{x}>0$ :

$$
\operatorname{Re}\left(\frac{\omega^{2} \mathrm{u}(\mathrm{i}+\mathrm{u})}{\beta(\mathrm{u})^{2}}\right) \leq-1 \wedge \operatorname{Im}\left(\frac{\omega^{2} \mathrm{u}(\mathrm{i}+\mathrm{u})}{\beta(\mathrm{u})^{2}}\right)=0
$$

Working out the condition on the imaginary part yields $\mathrm{xf}(\mathrm{x}, \mathrm{y}) /|\beta|^{2}=0$, where $\mathrm{f}$ was previously defined in (A.11). Since $|\beta|>0$ in case 2, and $x>0$ here, the condition boils down to:

$$
f(x, y)=\omega \rho(2 \kappa-\omega \rho)\left(x^{2}+y^{2}\right)+\kappa^{2}(1+2 y)=0
$$

Solving $f(x, y)=0$ for $x>0$ and inserting this in the first part of (A.25) yields:

$$
\frac{2 \kappa^{2}(\kappa-\omega \rho)^{2}\left((2 \kappa \rho-\omega) \kappa-\omega\left(\omega \rho+2 \kappa\left(1-2 \rho^{2}\right)\right) y-2 \omega^{2} \rho\left(1-\rho^{2}\right) y^{2}\right)}{\rho(2 \kappa-\omega \rho)^{2}} \leq 0
$$

We will now check that (A.26) and (A.27) cannot be simultaneously true for $\mathrm{x}>0$, by splitting the proof into the same regions we used in the proof of lemma A.4:

- $\quad \boldsymbol{\rho}<\mathbf{0}$ : (A.26) is true for $\mathrm{y} \leq \mathrm{y}_{1}$ and $\mathrm{y} \geq \mathrm{y}_{2}$. From case 1a) of lemma A.4 we know that for $\mathrm{y} \leq$ $\mathrm{y}_{1} \mathrm{f}$ is strictly increasing in $\mathrm{y}$, and strictly decreasing in $\mathrm{x}$. Furthermore, $\mathrm{f}\left(0, \mathrm{y}_{1}\right)<0$. Turning to $y \geq y_{2}$, case $\left.1 c\right)$ of the same lemma shows that $f$ is strictly decreasing in $y$, and strictly decreasing in $\mathrm{x}$. Furthermore, $\mathrm{f}\left(0, \mathrm{y}_{2}\right)=0$, which fully excludes (A.25) for $\mathrm{x}>0$ and $\rho<0$;

- $\rho=0$ : (A.27) is not well-defined for $\rho=0$. However, notice that for $x>0$ (A.26) can only be true if $y=-1 / 2$. Since $\beta=\kappa$ here, checking (A.25) yields:

$$
\operatorname{Re}\left(\frac{\omega^{2} \mathrm{u}(\mathrm{i}+\mathrm{u})}{\beta(\mathrm{u})^{2}}\right)+1=\frac{\kappa^{2}+\omega^{2}\left(\mathrm{x}^{2}+\frac{1}{4}\right)}{\kappa}>0
$$

- $\mathbf{0}<\rho \leq \kappa / \omega$ : For $\mathrm{y} \leq \mathrm{y}_{2}$ and $\mathrm{y} \geq \mathrm{y}_{1}$ (A.27) is again true. Case 3a) from lemma A.4 shows that $\mathrm{f}(\mathrm{x}, \mathrm{y})<0$ is true for $\mathrm{y} \leq \mathrm{y}_{2}$, and case $3 \mathrm{c}$ ) shows the same for $\mathrm{y} \geq \mathrm{y}_{1}$.

- $\kappa / \omega \leq \rho \leq 2 \kappa / \omega$ : We only checked this case for values of $y \geq y_{2}$, and (A.27) can only be true for $y \geq y_{1}$. Here, the analysis of case $\left.4 b\right)$ of lemma A.4 shows that $f(x, y)>0$ for $x>0$, except if $\mathrm{y}=\mathrm{y}_{1}$ and $\rho=2 \kappa / \omega$, when $\mathrm{f}\left(\mathrm{x}, \mathrm{y}_{1}\right)=0$ for any $\mathrm{x}>0$. 